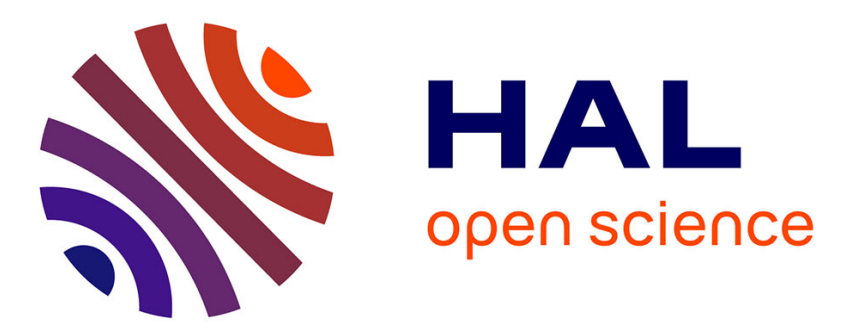

\title{
Modeling and controlling an isolated urban intersection based on cooperative vehicles
}

\author{
Mourad Ahmane, Abdeljalil Abbas-Turki, Florent Perronnet, Wu Jia, \\ Abdellah El-Moudni, Jocelyn Buisson, Renan Zéo
}

\section{- To cite this version:}

Mourad Ahmane, Abdeljalil Abbas-Turki, Florent Perronnet, Wu Jia, Abdellah El-Moudni, et al.. Modeling and controlling an isolated urban intersection based on cooperative vehicles. Transportation research. Part C, Emerging technologies, 2013, 28, pp.44-62. 10.1016/j.trc.2012.11.004 . hal00781330v3

\section{HAL Id: hal-00781330 \\ https://hal.inria.fr/hal-00781330v3}

Submitted on 4 Apr 2013

HAL is a multi-disciplinary open access archive for the deposit and dissemination of scientific research documents, whether they are published or not. The documents may come from teaching and research institutions in France or abroad, or from public or private research centers.
L'archive ouverte pluridisciplinaire HAL, est destinée au dépôt et à la diffusion de documents scientifiques de niveau recherche, publiés ou non, émanant des établissements d'enseignement et de recherche français ou étrangers, des laboratoires publics ou privés. 


\title{
Modeling and controlling an isolated urban intersection based on cooperative vehicles
}

\author{
Mourad Ahmane ${ }^{\mathrm{a}, *}$, Abdeljalil Abbas-Turki ${ }^{\mathrm{a}}$, Florent Perronnet ${ }^{\mathrm{a}}$, Jia Wu ${ }^{\mathrm{b}}$, Abdellah El \\ Moudni $^{\mathrm{a}}$, Jocelyn Buisson ${ }^{\mathrm{c}}$, Renan Zeo ${ }^{\mathrm{c}}$ \\ ${ }^{a}$ Laboratoire Systèmes et Transport, Université de Technologie de Belfort-Montbeliard, 90010 Belfort cedex, \\ France, www.utbm.fr \\ ${ }^{b}$ University of Electronic Science and Technology of China, www.oice.uestc.edu.cn/en \\ ${ }^{c}$ Voxelia, Belfort, France, www.voxelia.com
}

\begin{abstract}
In this paper, we propose a new approach for controlling the traffic at isolated intersections. We assume that all vehicles are equipped with on-board units (ITS station) that make them able to wirelessly negotiate the "right of way" according to the measurements done by the positioning system during their travel. A vehicle is allowed to cross the intersection if the green color is displayed to the driver in an on-board screen. The control aims to smooth the traffic through the sequence of vehicles authorized to traverse the intersection. The main challenge raised with the assumption is that the sequence must be dynamically formed by a real time application. The dynamic behavior of the traffic is considered discrete, in order to determine the switching rule according to the instantly observed events. We propose a model based on Timed Petri Nets with Multipliers (TPNM) which allows us to propose the control policy through the structural analysis. The resulting switching rules are very simplistic and efficient for isolated intersections. Indeed, microscopic simulations show that they perform as well as the optimal sequence based on the detection of vehicles at the entrance of the intersection. Moreover, the proposed approach has been tested through a real intersection.
\end{abstract}

Keywords:

Cooperative Intersection Management, Distributed real-time scheduling, Clearing policy, Timed Petri Net.

\section{Introduction}

Nowadays, as a consequence of population growth, motorization and urbanization, traditional urban traffic control strategies cannot meet the requirements of the modern city. Traffic

${ }^{*}$ Corresponding author, email: mourad.ahmane@yahoo.fr, Tel: +33.(0)3.84.58.30.38 
congestion takes place worldwide more frequently. It not only reduces the efficiency of transportation, but also increases air pollution and fuel consumption. This raises the issue of whether the supplied infrastructures correspond to the traffic demand. Invest in new infrastructures is more and more expensive. Hence, Intelligent Transportation Systems (ITS) are considered as the key for enhancing the capacity of the existing infrastructures. For instance, car platooning investigations (Contet and al., 2007a, 2007b; Ferrara and al., 2008; Pan, 2008) aim at increasing the capacity of roads by reducing temporal distances between vehicles (vehicles can move close together). In other words, researchers stake on new embedded and wireless communication technologies in order to improve traffic conditions. Contrasting with the penetration of communication technologies in several topics of the traffic engineering, investigations into the efficiency of the intersections are mainly based on the measurement of the magnetic loops (Henry and al., 1983; Chard and Lines, 1987; Robertson and Bretherton, 1991; Sims and Dobinson, 1980; Gartner and al., 2001; Motawej and al., 2011). This is contradicting with the fact that intersections are the area where city infrastructures are shared. In addition, intersections are mainly in the core of congestion. Such a lack of investigation about the communications at an intersection is due at least to these four following reasons:

1. Right now, intersection control decision involves only city authorities. Hence, vehicles cannot negotiate their access (Robertson and Bretherton, 1991; Sims and Dobinson, 1980, 2001; Henry and al., 1983).

2. We believe that it may be cheaper or faster to control vehicles as a flow than to process them individually (Potts and Kovaylov, 2000).

3. The problem of processing vehicles individually requires new rules of arbitration.

4. The traffic lights display the same sign to all vehicles that arrive from the same road.

Although these reasons are obviously very important, the traffic situation compels to change our point of view. Indeed, the states of the magnetic loop cannot accurately reflect the current traffic situation and thus we often face under-exploited infrastructures. For instance, one can meet cases where the vehicle can safely cross the intersection but the traffic light is red. Furthermore, developments of new technologies of communication and their democratization will let the control of vehicles individually cheaper and realizable. Recently, ITS stations are under development for making vehicles able to wirelessly communicate (Takikita and Goto, 2008). Hence, there is a recent research trend towards the decentralization of the traffic control, involving vehicles and embedded ITS stations in participating in the decision about the access to the intersection. This raises several issues such as finding an efficient communication protocol and well-adapted algorithms. The latter issue is the scope of this paper.

Let us recall some notable works. Naumann et al. (1997) have proposed a decentralized protocol for wirelessly negotiating the "right of way". They have assumed that vehicles are automatically driven and able to communicate with other vehicles. Each vehicle instantly computes its priority that evolves during the trajectory. Thus, it continuously sends the new 
computed priority to the surrounding vehicles. According to the received messages, the vehicle waits behind the stop line until all vehicles with a higher priority have crossed the intersection. Based on this protocol, Neuendorf and Bruns (2004) have introduced an approach for controlling the speed of vehicles. The protocol has been tested by using few mini robots (Grnewald and al., 2006). However, the test has revealed possible collisions.

An improvement of this protocol has been given by (Dresner and Stone, 2004). The simulations have shown that the wireless negotiation of the right of way contribute to improve traffic conditions at intersections. Regardless this fact, the approach requires an extraordinary precise control system of vehicle motions (Au and al., 2012), as it has been shown through the mixed reality platform on which a robot interacts with multiple virtual vehicles (Quinlan et al., 2010). Indeed, the protocol has raised the issue of the punctuality of the robot (De La Fortelle, 2010) since the "right of way" is a time and space reservation that the robot must respect. In simple words, there is a high risk of collision if a robot does not respect the reservation.

In order to overcome this drawback, new approaches have been proposed. According to the arrival times of vehicles, several algorithms compute the optimal sequence for distributing the "right of way". Indeed, sequences are easier to be respected than the time reservation. Li and Wang (2006) have presented an algorithm for reducing the evacuation time by evaluating all possible sequences. Wu and al. (2007) have introduced a dynamic programming able to obtain the optimal sequence. Yan and al. (2009) have compared this algorithm to a branch and bound algorithm. The simulations have shown that the proposed algorithms outperform traffic lights since they are more precise than the optimization of the duration of the green light given to a particular movement. Nevertheless, these algorithms raise the issue of the computing time for such a real-time application. Indeed, the decisions have to be taken during a very short time which corresponds to the cycle times of each embedded computer. Shorter than $1 s$, the cycle time is the duration of collecting data and of computing the appropriate decision. Moreover, the intersection system is dynamic. In other words, an optimal solution for a given time is not necessarily optimal if we consider the new arrivals of vehicles. A remarkable discussion of this problem is given in the introduction of the paper (Kumar and Seidman, 1990).

In this paper, a new control approach for urban intersection between four roads is proposed. The approach is based on the assumption that each vehicle is equipped with a positioning system and an ITS station for the wireless communication between vehicles and the infrastructure. In order to improve the computation performance required for the real-time control of the traffic, the work, presented in this paper, is based on the self-organization theory, that is simple and local rules lead to a global complex behavior. The scope of this paper is to provide efficient rules (control policy) for controlling an isolated intersection by considering the request messages, received from vehicles. The result of the control policy is a sequence of authorized vehicles, in which parallel and following vehicles can simultaneously obtain the "right of way".

From a theoretical point of view, the proposed control policy is based on the modeling of an isolated 4-ways intersection as a discrete event dynamic system. The dynamic behavior of 
the modeled traffic is discrete and represented by a Timed Petri Net with Multipliers (TPNM) in which multipliers are associated with the arcs of the Petri Net. From the TPNM model, constraints and linear system equations are derived in order to propose the control policy for distributing the "right of way". Because of the real-time decision making and lack of expectation (each vehicle is dealt individually), the objective of the control policy is to instantly minimize the queue length.

Such a real-time application raises the efficiency issue. Indeed, the performances of the proposed control policy must be as near as possible to the performances that result from the optimal sequence. Hence, we provide simulations for comparing the proposed control policy to the "global solution". The "global solution" is the optimal sequence of all simulated arrivals of vehicles. This sequence is computed according to the arrival times of vehicles as given in (Wu and al., 2007). The main objective of the "global solution" is to evacuate all simulated vehicles as soon as possible. The results of simulations show that the resulting performances of both controls are comparable. Moreover, the proposed approach has been tested through a real intersection of ordinary vehicles.

The paper is organized as follows. Section 2 presents the treated problem and describes the studied system. It is modeled by means of a TPNM, in Section 3. A structural analysis of the model is given in the same section. Section 4 is devoted to give and implement the control policy for the traffic of cooperative vehicles at an isolated intersection. Moreover, an example is given to illustrate the control policy. In Section 5, we describe the experiments we have done through a real cooperative intersection for testing the proposed approach. We give also some simulation results in order to assess the control policy. Finally, we conclude in Section 6.

\section{Problem Description}

We would like to remind readers that one of the main objectives of this paper is to explore a new traffic control concept and to provide the corresponding theoretical basis for it. Therefore, only a simple intersection model is considered. However, the control policy mentioned in this paper can be applied to other isolated intersections with different layouts.

To achieve the control objectives, the problem needs to be abstracted into a mathematical form. Firstly, we consider an isolated intersection with a novel signaling system as showed in Figure 1.

We recall that each vehicle is equipped with an ITS station which permits to communicate with the other vehicles through a server. When a vehicle arrives at the intersection, it instantly requests the "right of way", until it gets it. The negotiation consists in sending its position during its trajectory. We assume that each vehicle is equipped with an on-board signalization to indicate the state of the "right of way". There are only two colors of signalization displayed to the driver, i.e., green (with the "right of way") and red (without "right of way"). The yellow could exist for indicating failures of the system or other dangerous cases such as stopped 


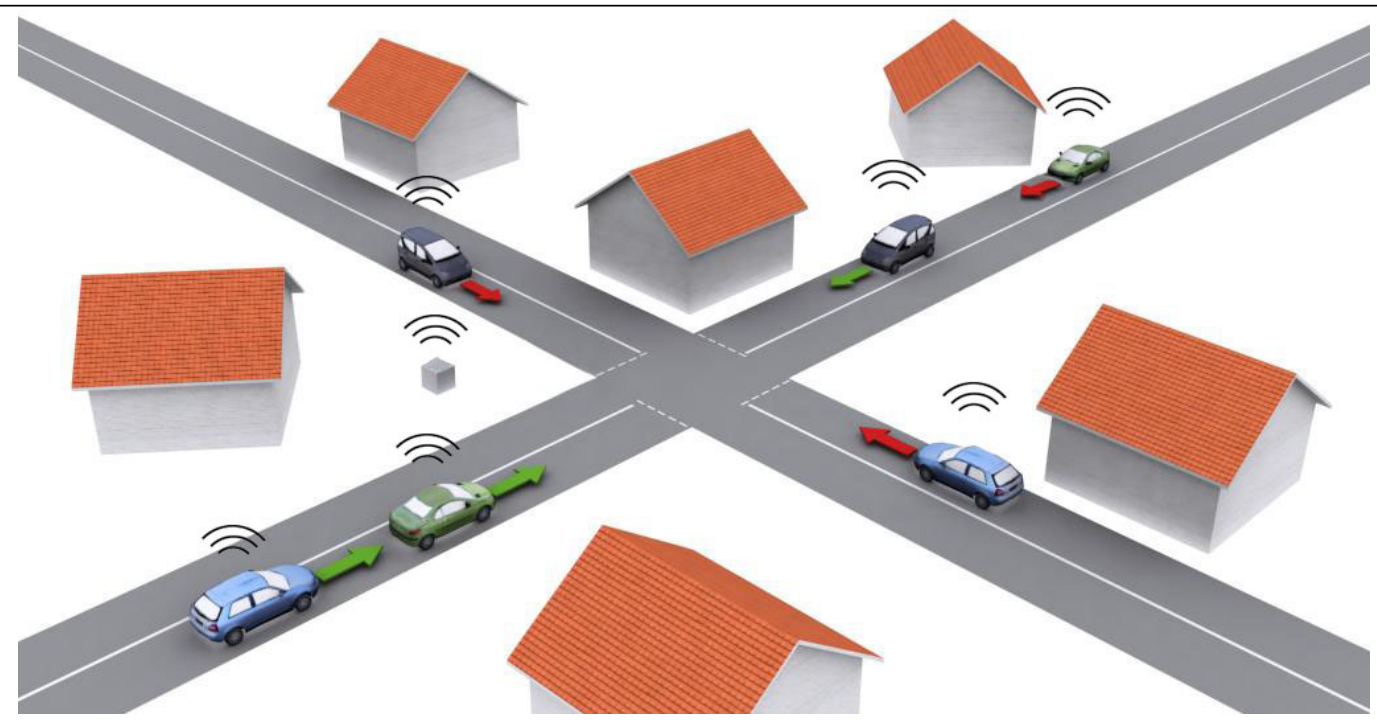

Figure 1: Isolated Intersection with cooperative vehicles

vehicles at the next intersection (De la fortelle, 2010). But these issues are not treated in this paper.

Figure 2 shows the studied isolated intersection in an abstracted manner. Indeed, the server that controls the access to the intersection is removed because it is considered as a support of a reliable negotiation of the "right of way" between vehicles and not as a scheduler. We note that there are two conflicting traffic flows to be served in this intersection, i.e. go-straight traffic flow of direction West-East $(F 1)$ and go-straight traffic flow of direction North-South $(F 2)$.

With regard to our intersection, we assume that:

1. Each vehicle is equipped with an ITS station which permits to communicate with other vehicles at the intersection through a server.

2. Vehicles in the same road cross the intersection in the order of First In First Out (FIFO).

3. There is a minimum time space $S$ between two successive vehicles coming from two conflicting traffic flows. The time space $S$ includes the time that each vehicle uses to cross the intersection, the acceleration time and the security time space. Practically, $S$ depends on the statistics of vehicles and the layout of the studied intersection.

4. Two successive vehicles in the same road are spaced at least by a time space $d$ when they cross the conflict zone: $d<S$. This security time distance is decided by the minimum driver reaction time and the mechanical and hydraulic systems to respond. A considerable amount of research has been dedicated to the analysis of the headway time (Kanaris and al., 1997). 


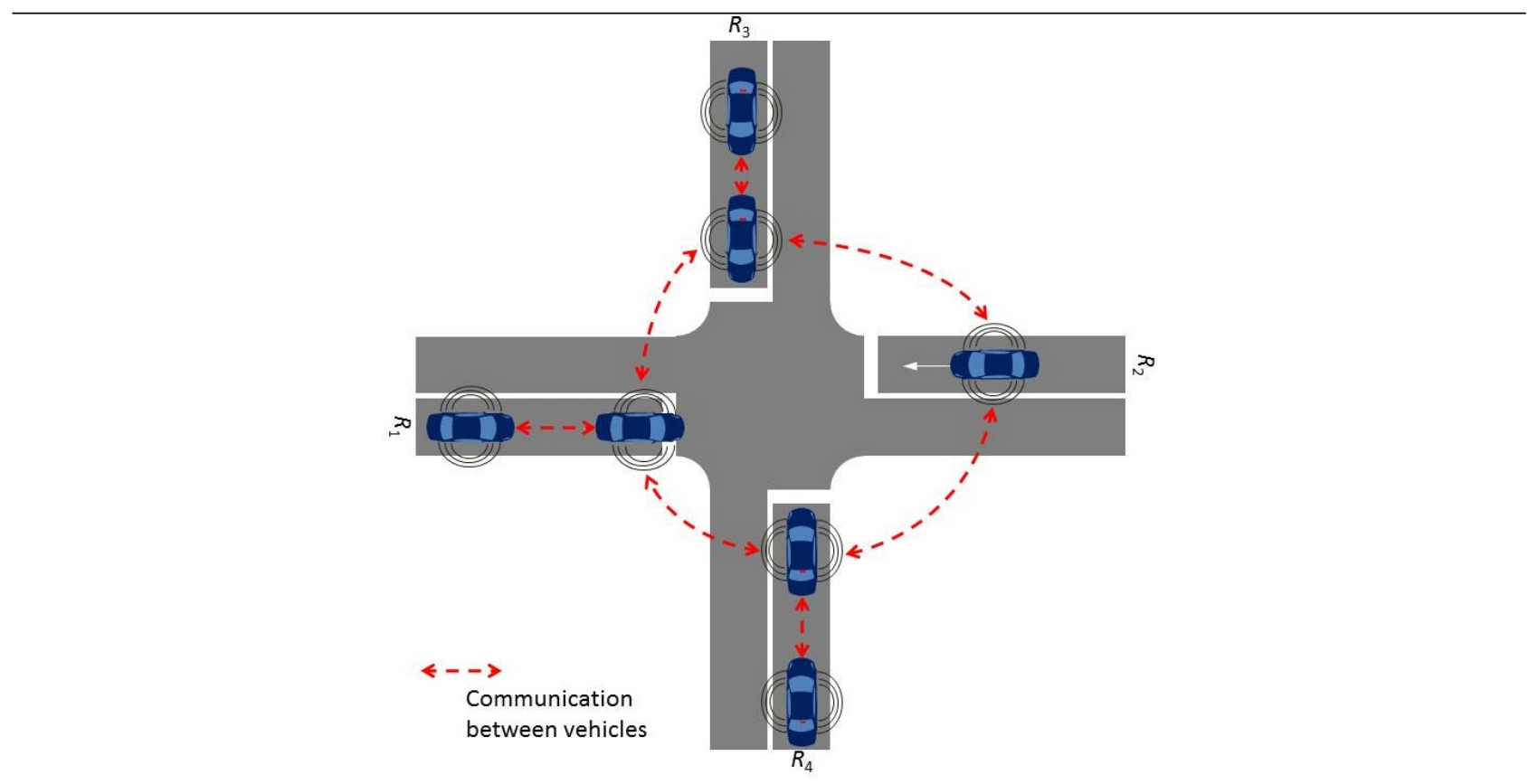

Figure 2: Negotiation of the "right of way" between cooperative vehicles

5. A vehicle whose distance to the precedent vehicle is less than or equal to $d$ constitutes a "team" with the vehicle in front. For instance, in Figure 2 the first two vehicles in road $R_{4}$ constitute a "team of vehicles".

6. The "rights of way" are wirelessly communicated to the vehicles. Two vehicles that move in two conflicting traffic flows cannot get simultaneously the "right of way".

7. The default color of the on-board signalization is red (default deny).

8. If a vehicle has obtained the "right of way", it keeps it until it leaves the intersection.

Secondly, the detection of vehicle in the intersection is obtained when they pass over the position markers located on roads, as shown in Figure 3.

This system considers four roads. Each road has three position markers. Since the conflict zone is the core of the problem, we will focus on the sequence of the observed events at the position marker 2. This brings us to treat individually each vehicle. We use discrete timed Petri net modeling to tackle our problem.

\section{Modeling with Petri Nets}

The idea of applying Petri Nets (PN) to model Traffic Networks can be dated back to the approach presented in (Giua, 1991). In (Di Febbraro and al., 2004), a model of an urban 


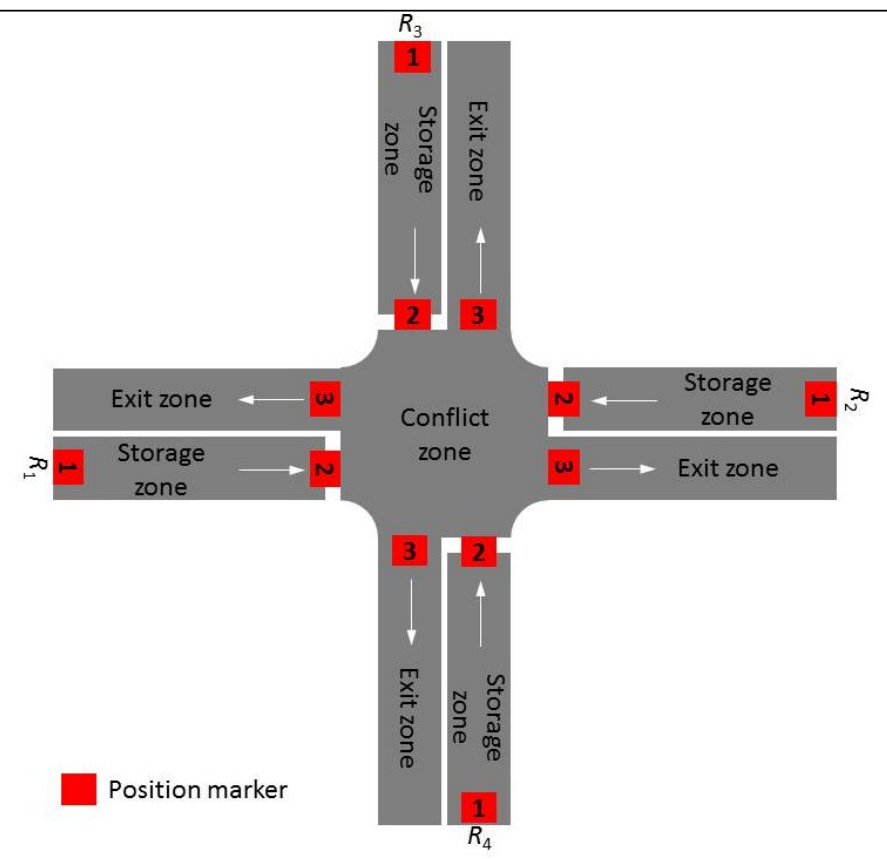

Figure 3: Position markers

network of signalized intersections via hybrid PN has been used to state and solve the problem of coordinating several traffic lights with the aim of improving the performances of some classes of special vehicles, i.e., public and emergency vehicles. In (Bouyekhf and al., 2003; Jingyu and Qiqiang, 2008), a Dynamic Stochastic Fluid PN has been proposed which can give a good description of the dynamic and stochastic character of the urban traffic system. Dotoli and Fanti (2006) have proposed a modular framework based on colored timed PN to model the dynamics of signalized traffic network systems where places represent link cells and crossing sections, tokens are vehicles and token colors represent the routing of the corresponding vehicle.

\subsection{Preliminaries}

PN have proven to be extremely useful in modeling, analysis, control and performance evaluation for a large scale of discrete event systems (Murata, 1989). Formally, a PN is a five-tuple $N=\left(P, T, I, O, M_{0}\right)$ where:

- $P=P_{1}, P_{2}, \ldots, P_{m}$ is a finite set of places (represented by circles),

- $T=T_{1}, T_{2}, \ldots, T_{n}$ is a finite set of transitions (represented by line segments),

- $I: P \times T \rightarrow \mathbb{N}$, is the input function such that $I\left(P_{i}, T_{j}\right)$ is the weight of the arc directed from $P_{i}$ to $T_{j}$, 
- $O: P \times T \rightarrow \mathbb{N}$, is the output function such that $O\left(P_{i}, T_{j}\right)$ is the weight of the arc directed from $T_{j}$ to $P_{i}$,

- $M_{0}$ is an initial marking that associates zero or more tokens to each place.

Furthermore, $n \geq 0, m \geq 0, n+m \geq 1$ and $P \cap T=\emptyset$.

The state of a PN is defined by the number of tokens in each place and is represented by a vector $M=\left[M\left(P_{1}\right), M\left(P_{2}\right), \ldots, M\left(P_{m}\right)\right]^{T}$, called the marking vector of the PN, where $M\left(P_{i}\right)$ is the number of tokens in a place $P_{i}$. A transition $T_{j} \in T$ is said to be enabled if and only if $M\left(P_{i}\right) \geq I\left(P_{i}, T_{j}\right), \forall P_{i} \in P$. An enabled transition may fire. When a transition $T_{j}$ fires, a token is removed from each of its input places and a token is added to each of its output places.

The dynamic behavior of the modeled system is described by the transitions firing mechanism. If the transition $T_{j}$ is fired then the marking $M_{0}\left(P_{i}\right)$ results in new marking $M\left(P_{i}\right)$ such that:

$$
M\left(P_{i}\right)=M_{0}\left(P_{i}\right)+O\left(P_{i}, T_{j}\right)-I\left(P_{i}, T_{j}\right) .
$$

Let $R\left(M_{0}\right)$ denotes the set of all markings reachable from $M_{0}$. The incidence matrix $C$ of a Petri net is an $m \times n$ matrix of integers defined as $C=O-I$, where $C_{i j}=O\left(P_{i}, T_{j}\right)-I\left(P_{i}, T_{j}\right)$. The marking $M \in R\left(M_{0}\right)$ is reached when a firing sequence $x$ is executed from $M_{0}$ and satisfies the state equation:

$$
M=M_{0}+C x,
$$

where $x: T \rightarrow \mathbb{N}$ is the firing vector sequence given by $x=\left[x_{1}, \ldots, x_{n}\right]^{\top}$, where $x_{j}$ is the number of times that $T_{j}$ is fired.

An event graph is a PN such that each place has only one input arc and one output arc. If the tokens have to stay a minimum amount of time in the places, we speak of Timed Event Graph (TEG).

A Timed PN with Multipliers (TPNM) is a Timed PN with natural numbers associated to arcs (multipliers) in order to model a wide range of systems. For example, when the achievement of a specific task requires several units of a same resource, or when an assembly operation requires several units of a same part.

\subsection{The TPNM model of the intersection}

We assume that the four roads are similar. The Timed Petri Net with Multipliers (TPNM) model of the isolated intersection of Figure 2 is given in Figure 6. Before that, we try to explain in detail the three different components: The sub-model $\left(R_{1}, R_{2}\right)$, the sub-model $\left(R_{3}, R_{4}\right)$ and the sub-model (Traffic control). The sub-model traffic control represents the distribution of the "right of way" to the different vehicles. This model prevents two vehicles that come from each pair of conflicting roads from simultaneously crossing the conflict zone. 


\subsection{1. $\operatorname{Road}\left(R_{i}\right)$}

In the following, we give the Timed Petri Net model of a road $R_{i}(i=1,4)$ of Figure 2. This model without considering any traffic control is given into Figure 4 . The meanings of each transition is given in Table 1.

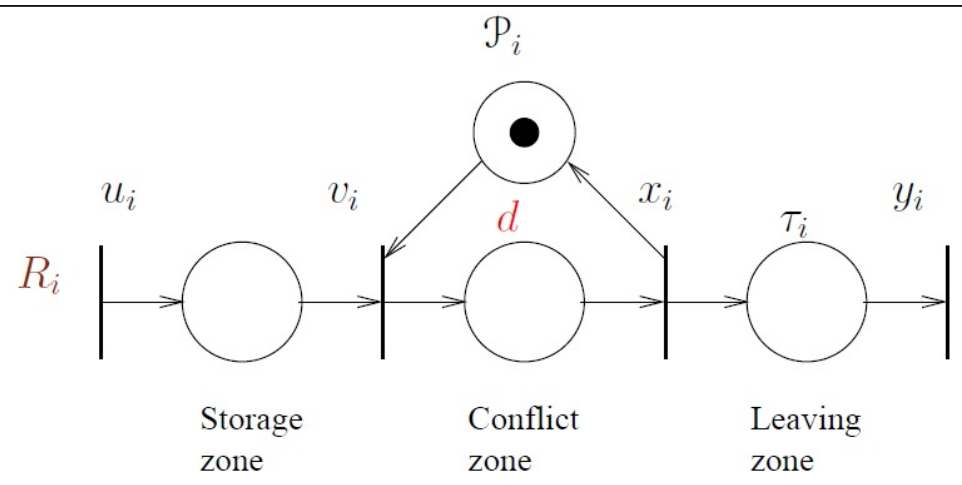

Figure 4: Road $R_{i}$

\begin{tabular}{|c||l|}
\hline Transition & Meaning \\
\hline \hline$u_{i}(t)$ & $\begin{array}{l}\text { - Number of vehicles ready to cross the intersection from } R_{i} \text { (or have passed } \\
\text { the position marker } 1 \text { ) until time } t .\end{array}$ \\
$u_{i}(k)$ & - Time when the $k^{t h}$ vehicle of $R_{i}$ is ready to cross the intersection. \\
\hline$v_{i}(t)$ & - Number of vehicles that accessed to the conflict zone from $R_{i}$ (or have passed \\
& the position marker 2) until time $t$. \\
$v_{i}(k)$ & - Time when the $k^{t h}$ vehicle of $R_{i}$ accessed to the conflict zone. \\
\hline$x_{i}(t)$ & $\begin{array}{l}\text { - Number of vehicles that have left the conflict zone from } R_{i} \text { (or have passed } \\
\text { the position marker } 3) \text { until time } t .\end{array}$ \\
$x_{i}(k)$ & $\begin{array}{l}\text { - Time when the } k^{t h} \text { vehicle of } R_{i} \text { allows other vehicles to pass through the } \\
\text { conflict zone. }\end{array}$ \\
\hline
\end{tabular}

Table 1: Meanings of transitions of Road $R_{i}$

- Place $\mathcal{P}_{i}$ is timed to hold the minimum security time distance $d$ between two successive vehicles when they cross the conflict zone.

- Time $d$ means that two successive vehicles in the same road (from the same traffic flow) are spaced at least by a time space $d$ when they cross the conflict zone (shared resource).

- Except for Place $\mathcal{P}_{i}$, the presence of a token in each place means that a vehicle crosses the concerned zone. 
- Time $\tau_{i}$ corresponds to the complete time needed for passing the intersection.

\subsubsection{The traffic control}

The traffic control represents the state of the signalization. In the following, we discuss the TPNM model given in Figure 5. The meanings of each transition is given in Table 2.

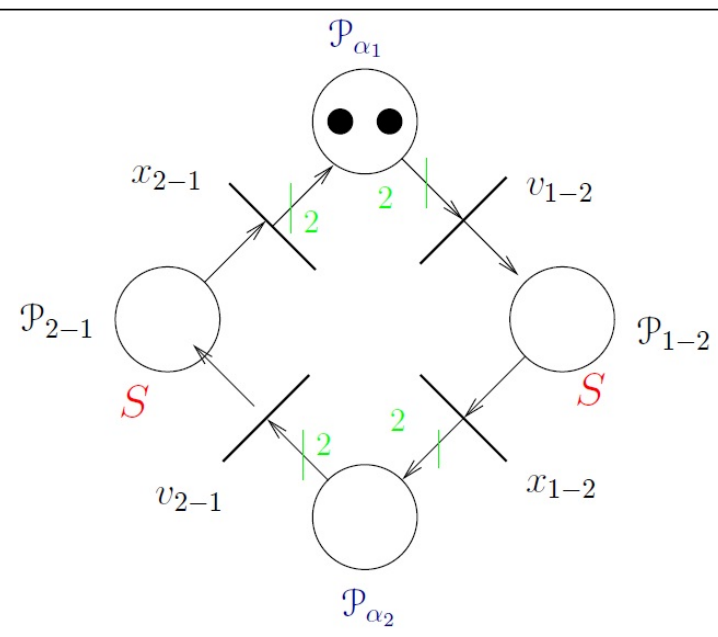

Figure 5: Traffic control

\begin{tabular}{|c||l|}
\hline Transition & Meaning \\
\hline \hline$v_{i-j}$ & The beginning of the security time $S$. \\
\hline$x_{i-j}$ & The end of the security time $S$. \\
\hline
\end{tabular}

Table 2: Meanings of transitions of the traffic control

- Place $\mathcal{P}_{\alpha_{i}}$ is an immediate place (without temporization, fired immediately) which allows vehicles moving in the same traffic flow $F i$ to cross the conflict zone at the same time. $\mathcal{P}_{\alpha_{1}}$ allows to move in roads $R_{1}$ and $R_{2}(F 1)$ at the same time and $\mathcal{P}_{\alpha_{2}}$ allows to move in roads $R_{3}$ and $R_{4}(F 2)$ at the same time.

- The presence of two tokens in place $\mathcal{P}_{\alpha_{1}}$ means that the "right of way" is given to vehicles that move in roads $R_{1}$ and $R_{2}$ simultaneously.

- Place $\mathcal{P}_{i-j}$ is timed in order to hold the token and thus to model the security time $S$ when the "right of way" switches from $F i$ to $F j$, where $i \neq j$ and $i, j \in 1,2$. 
- Time $S$ represents the minimum security time distance between two successive vehicles in $F i$ and $F j$.

- The Multipliers 2 on arcs means that weights 2 are added to the corresponding arcs. For instance, transition $v_{1-2}$ can be fired only if there are two tokens available in the input place $\mathcal{P}_{\alpha_{1}}$. If we enable transition $v_{1-2}$ we should remove two tokens from the input place $\mathcal{P}_{\alpha_{1}}$ and add only one token to the output place $\mathcal{P}_{1-2}$. On the other hand, If we enable transition $x_{1-2}$ we should remove one token from the input place $\mathcal{P}_{1-2}$ and add two tokens to the output place $\mathcal{P}_{\alpha_{2}}$. This aims to prevent two vehicles that move in conflicting roads from simultaneously getting the "right of way".

\subsubsection{TPNM model}

Since the intersection of Figure 2 is composed by the three different components: The submodel $\left(R_{1}, R_{2}\right)$, the sub-model $\left(R_{3}, R_{4}\right)$ and the sub-model (Traffic control), by concatenation of the two components defined by Figure 4 and Figure 5 we obtain the Timed Petri Net with Multiplier (TPNM) depicted in Figure 6. The meanings of each transition is given in Table 3.

\begin{tabular}{|c||l|}
\hline Transition & Meaning \\
\hline \hline$u_{i}(t)$ & $\begin{array}{l}\text { - Number of vehicles ready to cross the intersection from } R_{i} \text { (or have passed } \\
\text { the position marker } 1 \text { ) until time } t .\end{array}$ \\
$u_{i}(k)$ & - Time when the $k^{t h}$ vehicle of $R_{i}$ is ready to cross the intersection. \\
\hline$v_{i}(t)$ & $\begin{array}{l}\text { - Number of vehicles that accessed to the conflict zone from } R_{i} \text { (or have passed } \\
\text { the position marker } 2 \text { ) until time } t .\end{array}$ \\
$v_{i}(k)$ & - Time when the $k^{t h}$ vehicle of $R_{i}$ accessed to the conflict zone. \\
\hline$x_{i}(t)$ & $\begin{array}{l}\text { - Number of vehicles that have left the conflict zone from } R_{i} \text { (or have passed } \\
\text { the position marker } 3) \text { until time } t .\end{array}$ \\
$x_{i}(k)$ & $\begin{array}{l}\text { - Time when the } k^{t h} \text { vehicle of } R_{i} \text { allows other vehicles to pass through the } \\
\text { conflict zone. }\end{array}$ \\
\hline$v_{i-j}$ & The beginning of the security time $S$. \\
\hline$x_{i-j}$ & The end of the security time $S$. \\
\hline
\end{tabular}

Table 3: Meanings of transitions of the TPNM model

The model has three parts, separated by two dashed lines. The three parts are traffic flow $F 1$, traffic control and traffic flow $F 2$. $F 1$ represents the states of roads $R_{1}$ and $R_{2}$. F2 represents the states of roads $R_{3}$ and $R_{4}$. The traffic control model represents the states of the signalization. Places $\mathcal{P}_{1}, \mathcal{P}_{2}, \mathcal{P}_{3}$ and $\mathcal{P}_{4}$ represent the required time space distance $d$ between two successive vehicles from the same road. Places $\mathcal{P}_{1-2}$ and $\mathcal{P}_{2-1}$ represent the switching of the traffic signalization. The tokens in place $\mathcal{P}_{\alpha_{i}}$ cannot allow the firing of the transitions $\left(v_{1}, v_{2}\right)$ and $\left(v_{3}, v_{4}\right)$ simultaneously. Place $\mathcal{P}_{i-j}$ is timed in order to hold the token and thus 


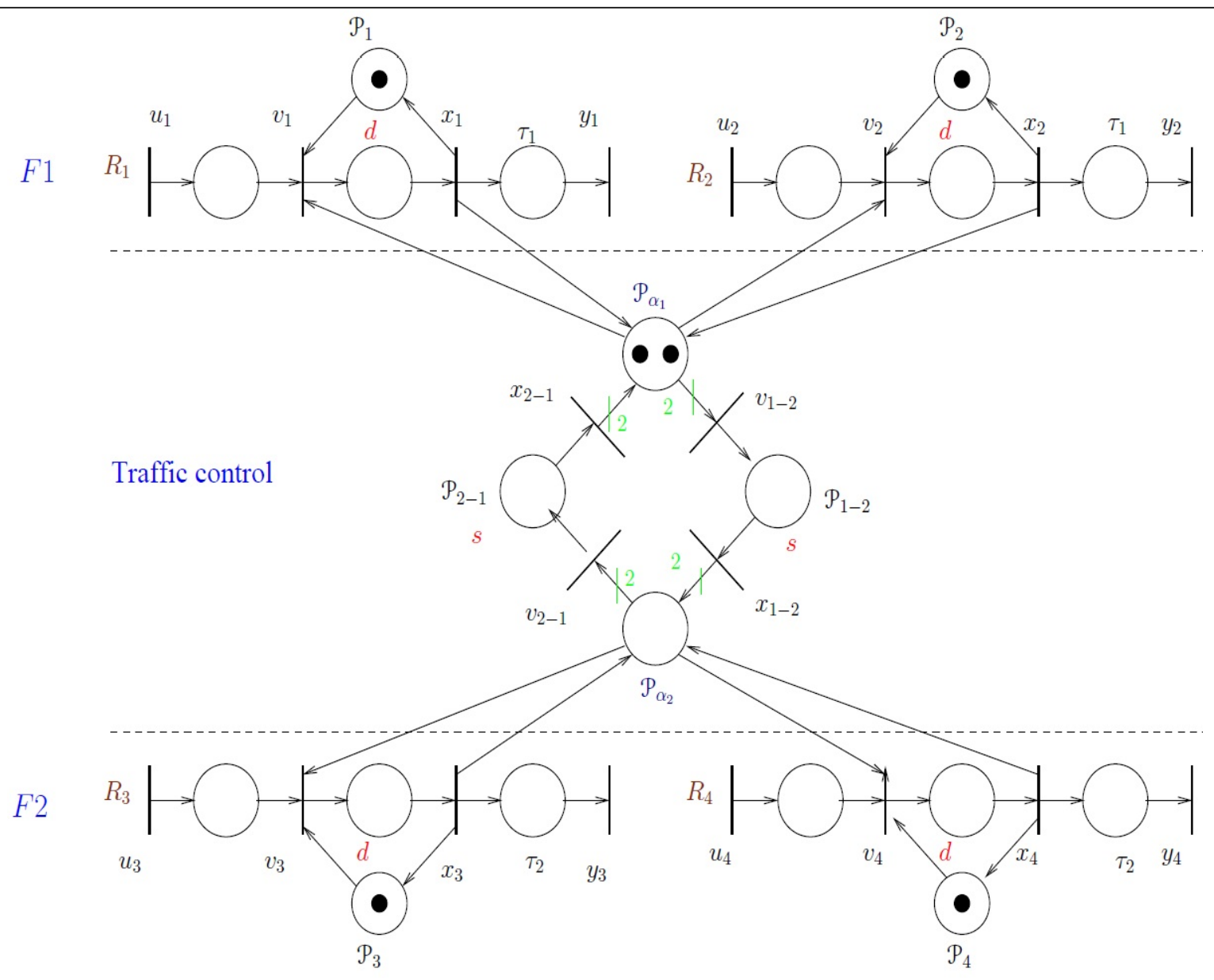

Figure 6: TPNM model of the intersection with traffic control

to model the security time when the authorization to pass through the intersection changes from $F i$ to $F j$, where $i, j \in 1,2$. $S$ represents the minimum security time distance between two successive vehicles in $F i$ and $F j$. We remark that there are two conflicts, the first one is between transitions $v_{1}, v_{2}$ and $v_{1-2}$ and the second between $v_{3}, v_{4}$ and $v_{2-1}$. The problem is to determine the sequence of firing of these transitions.

\subsection{Structural analysis}

Since the PN model of the system without intersection is a Timed Event Graph (TEG), the TPNM model of Figure 6 is divided into two sub-PN as clarified by Figure 7 .

- The first sub-PN gathers TEGs representing the vehicles in the roads before the stop line. 


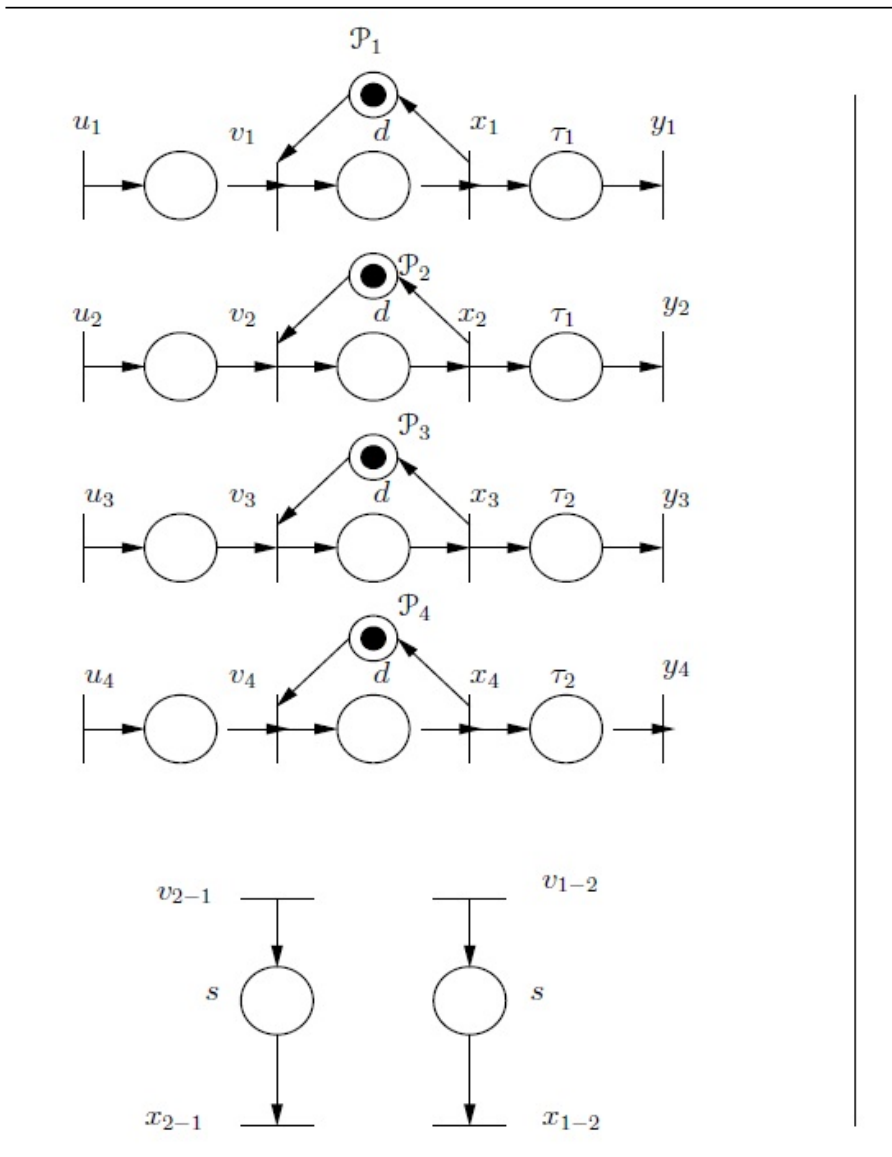

(a) TEG part
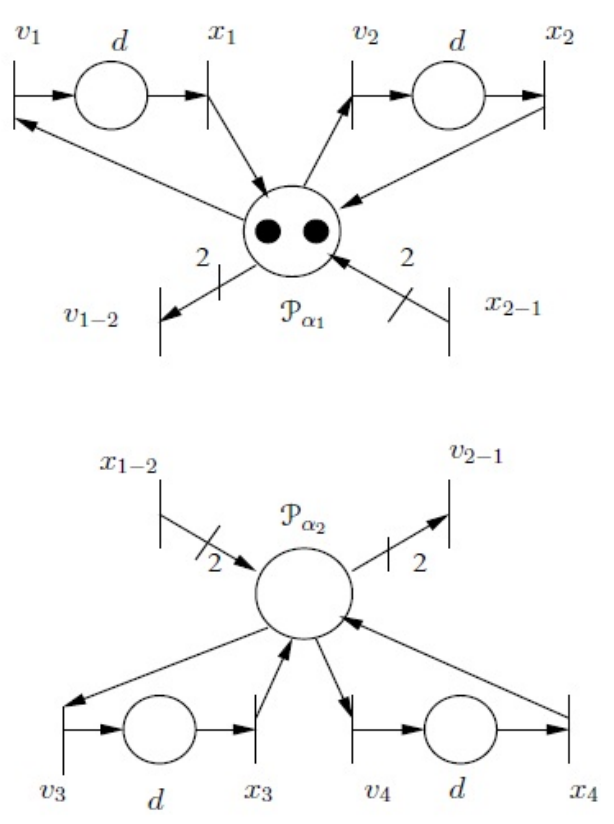

(b) PN part denoted $\zeta_{\alpha}$

Figure 7: Sub-PNs

- The second sub-PN represents the vehicles in the conflict zone.

In order to study the behavior of the vehicles evolving in the intersection, transitions $v$ and $x$ are duplicated. This allows us to simplify the analysis of the intersection by eliminating the external influence of the roads. Thus we consider $\zeta_{\alpha}$ as the model of shared resources. The structural analysis of $\zeta_{\alpha}$ provides us with Lemma 1.

Lemma 1 (Constraints). Let us consider Figure 7. Taking the resource $P_{\alpha_{i}}$. At each time $t$, the input and output transition counters $v_{i}(t)$ and $x_{i}(t)$, as well as the transition counters reflecting beginning and the end of configuration of the resource $P_{\alpha_{i}}$, noted respectively $x_{j-i}(t)$ 
and $v_{i-j}(t)$, satisfy the following inequalities:

$$
\sum_{j=2 i-1}^{2 i}\left\{v_{j}(t)-x_{j}(t)\right\}+\sum_{j \neq i, j=1}^{n}\left\{M_{0}\left(P_{\alpha_{i}}\right) \times v_{i-j}(t)-M_{0}\left(P_{\alpha_{i}}\right) \times x_{j-i}(t)\right\} \leq M_{0}\left(P_{\alpha_{i}}\right),
$$

with $n$ is the number of shared resources $P_{\alpha_{i}}$ and $M_{0}\left(P_{\alpha_{i}}\right)$ the initial marking of places $P_{\alpha_{i}}$.

Proof. By definition, the transitions $v_{i}$ and $v_{i-j}, \forall i$, are validated if and only if there are at least two tokens in the corresponding place $P_{\alpha_{i}}$. Using equations (1) and (2), the instantaneous marking of the place $P_{\alpha_{i}}$ is given as follows:

$$
\begin{aligned}
M_{t}\left(P_{\alpha_{i}}\right)= & M_{0}\left(P_{\alpha_{i}}\right)+\sum_{j=2 i-1}^{2 i} x_{j}(t)+\sum_{i \neq j, i=1}^{n} M_{0}\left(P_{\alpha_{i}}\right) \times x_{i-j}(t) \\
& -\sum_{i \neq j, i=1}^{n} M_{0}\left(P_{\alpha_{i}}\right) \times v_{j-i}(t)-\sum_{j=2 i-1}^{2 i} v_{j}(t) .
\end{aligned}
$$

But by definition: $M_{t}\left(P_{\alpha_{i}}\right) \geq 0 \forall t$, then the lemma is proved.

Remark 1. It is important to note that Lemma 1 is a very powerful means to give constraints of the studied system. Indeed, it provides us with a relation which implies only counters of transitions involved in $\zeta_{\alpha}$ and the number of initial free resources. However, from both a theoretical and a practical point of view, the constraint (3) permits to verify that:

(i) the constraint denies the request for a resource if there is no free resource,

(ii) no transition $v_{i}$ can be fired despite the lack of a free resource,

(iii) no transition $v_{i-j}$ can be fired despite the lack of two free resources. In other words, a transition $v_{i-j}$ is allowed to be fired if there are two free resources.

\section{The control strategy}

As described before, we assume that all vehicles have an embedded device that allows them to communicate their position as shown in Figure 2.

Let us recall that, in the literature, the objective is to evacuate all vehicles waiting on the roads as soon as possible (increase the throughput). However, the control is still based on a centralized coordination, while our objective is based on the self-organization theory, in order to fulfill the conditions of the real-time application. More precisely, we have to find simple and local rules that lead to a global complex behavior. Thus, the objective function must be instantly treated. The sum of queue lengths is an interesting way to tackle the matter. Indeed, there is a strong relation between throughput, delays and the queue lengths. For more detail about this relation, see the introduction of (Papageorgiou and al, 2003). 
The cooperative vehicle's scenario distributes the authority to each vehicle according to a particular protocol. This scenario gives autonomy to vehicles where they actually contribute to the decision whether or not they cross the intersection. In order to find a satisfactory way to control the intersection we use the mathematical model that describes the behavior of the TPNM presented in Figure 6. We can write the conflicting part of the model given in Figure 6 as follows:

$$
\left\{\begin{array}{l}
\forall i=1,2,3,4,\left\{v_{i}(t), u_{i}(t), v_{i-j}(t), x_{i}(t), x_{i-j}(t)\right\} \geq 0 \\
v_{i}(t) \leq u_{i}(t) \\
v_{1}(t)+v_{2}(t)+\left(2 \times v_{1-2}(t)\right) \leq 2+x_{1}(t)+x_{2}(t)+\left(2 \times x_{2-1}(t)\right), \\
v_{3}(t)+v_{4}(t)+\left(2 \times v_{2-1}(t)\right) \leq x_{3}(t)+x_{4}(t)+\left(2 \times x_{1-2}(t)\right), \\
x_{1}(t)=v_{1}(t-d), x_{2}(t)=v_{2}(t-d), x_{3}(t)=v_{3}(t-d), x_{4}(t)=v_{4}(t-d), \\
x_{1-2}(t)=v_{1-2}(t-S), x_{2-1}(t)=v_{2-1}(t-S) .
\end{array}\right.
$$

Remark 2. One can note from the system of constraints (5), that there is no vehicle in the intersection for $t<0$, as it is showed in the model given in Figure 6. For the sake of readability, and without loss of generality, this consideration will be kept in the remainder of the paper as well as the assumption stating that all transition counters are null for $t<0$. In other words, there is no transition fired before $t=0$.

\subsection{The study of the events}

Let $q_{1}(t), q_{2}(t), q_{3}(t)$ and $q_{4}(t)$ denote the number of vehicles waiting in Roads $R_{1}, R_{2}, R_{3}$ and $R_{4}$ at time $t$, respectively. Consider the function $J$ which represents the total number of vehicles waiting on all roads defined by $J=q_{1}(t)+q_{2}(t)+q_{3}(t)+q_{4}(t)$ for any time $t$. The choice of $J$ assumes that all roads have the same weight because the control strategy is not based on the past measurements of the traffic rates and priority roads. We draw the reader attention to the fact that we will not consider an upper bound of queue lengths because this work focuses on an isolated intersection. The lower bound is included in the second inequality of the system of constraints (5).

Let us recall that the objective of the paper is to instantly reduce the queue length when vehicles approach the intersection. In other words, the objective is to minimize the total number of cars waiting. It returns in minimizing the objective function $J$. The following proposition gives us an interesting property of the optimal solution to minimize $J$.

Proposition 2. Under the condition that in each served road there is at least one vehicle, the optimal solution of $\min _{t} J(t)$ minimizes the switching of the "right of way" between two conflicting traffic flows $F 1$ and $F 2$.

Proof. Let $q_{1}(t), q_{2}(t), q_{3}(t)$ and $q_{4}(t)$ denote the number of vehicles waiting in roads $R_{1}, R_{2}$, $R_{3}$ and $R_{4}$, respectively. The control objective is to minimize the total number of cars waiting on all roads, i.e. to minimize $J=q_{1}(t)+q_{2}(t)+q_{3}(t)+q_{4}(t)$ for any time $t$. 
Let us consider that the TPNM model depicted in Figure 6 is functioning at maximal speed. In other words, if an authorized road is empty, a switching is enforced but the conflicts still exist when in each road, there is at least one vehicle. Formally, the third and forth inequalities given in (5) become equalities. Thus, from the third inequality we have:

$$
v_{1}(t)+v_{2}(t)+2 \times v_{1-2}(t)=2+v_{1}(t-d)+v_{2}(t-d)+2 \times v_{2-1}(t-S) .
$$

According to the definition $q_{i}(t)=u_{i}(t)-v_{i}(t)$ for $i=1,2$. Replace it in (6) we obtain:

$$
\begin{aligned}
2= & u_{1}(t)-q_{1}(t)+u_{2}(t)-q_{2}(t)-u_{1}(t-d) \\
& +q_{1}(t-d)-u_{2}(t-d)+q_{2}(t-d)+2 \times\left(v_{1-2}(t)-v_{2-1}(t-S)\right) .
\end{aligned}
$$

Since $\Delta q_{i}(t)=q_{i}(t)-q_{i}(t-d), \Delta u_{i}(t)=u_{i}(t)-u_{i}(t-d), i=1,2$, equation (7) can be written as:

$$
2=\Delta u_{1}(t)-\Delta q_{1}(t)+\Delta u_{2}(t)-\Delta q_{2}(t)+2 \times\left(v_{1-2}(t)-v_{2-1}(t-S)\right) .
$$

From remark 2, note that $q_{i}(t)=\sum_{j=0}^{[t / d]} \Delta q_{i}(t-j \times d)$ and $u_{i}(t)=\sum_{j=0}^{[t / d]} \Delta u_{i}(t-j \times d)$ with $i=1,2$, in which $[x]$ is the integer part of $x$. We have from equation (8), the following equality:

$$
q_{1}(t)+q_{2}(t)=u_{1}(t)+u_{2}(t)+2 \times\left(\sum_{j=0}^{[t / d]}\left(v_{1-2}(t-j \times d)-v_{2-1}(t-S-j \times d)\right)-[t / d]-1\right) .
$$

The same analysis process for roads $R_{3}$ and $R_{4}$ can be applied to the fourth inequality of (5):

$$
q_{3}(t)+q_{4}(t)=u_{3}(t)+u_{4}(t)+2 \times\left(\sum_{j=0}^{[t / d]}\left(v_{2-1}(t-j \times d)-v_{1-2}(t-S-j \times d)\right) .\right.
$$

Hence, the objective function $J$ becomes:

$$
\begin{aligned}
J=\sum_{i=1}^{4} q_{i}(t)= & \sum_{i=1}^{4} u_{i}(t)+2 \times\left(\sum _ { j = 0 } ^ { [ t / d ] } \left(\left(v_{1-2}(t-j \times d)-v_{1-2}(t-S-j \times d)\right)\right.\right. \\
& \left.+\left(v_{2-1}(t-j \times d)-v_{2-1}(t-S-j \times d)\right)-[t / d]-1\right),
\end{aligned}
$$

Since $v_{1-2}(t)$ and $v_{2-1}(t)$ are monotonic increasing sequences because both $v_{1-2}(t)$ and $v_{2-1}(t)$ are counter functions, each firing of $v_{1-2}$ and $v_{2-1}$ increases the objective function $J$. $\square$

Remark 3. Proposition 2 shows that the switching of the "right of way" must be avoided as possible as we can. Hence, we have here an interesting property of the optimal solution in a general case. From practical point view, according to the kept criteria, the proposition informs 
us that it is better to continue the green period for the following vehicle when the time distance between two successive vehicles from a road $R_{i}$ is less than or equal to $d(\leq d)$ with $i=1,2,3,4$. This is true for the symmetric case in which either in each served road there is at least one vehicle ready to cross the intersection or both parallel roads are empty. In the following, we extend the rule to all cases, since the field of view of the controller is limited to $t$ (present time). Indeed, from the same analysis given in the proof, one can easily obtain that at each $t, J$ is incremented by the number of roads in which we refuse to continue authorizing the following vehicle.

\subsection{Implementation of the control strategy}

From Proposition 2, we give the following abstracted approach (without considering the server): Vehicles from the same traffic flow communicate with its neighbor, the vehicle just behind. By communication, each vehicle whose distance to the front vehicle is less than or equal to $d$ constitutes a team with the vehicle in front. For instance, the first two vehicles in road $R_{4}$ of Figure 8 constitute a team. The vehicles in the same team pass the intersection together without interruption.

Since the Timed Petri Net model supposes that the intersection is always occupied by vehicles or in the state of integral red light, it does not consider the case that the interval between two neighbor vehicles in both lanes are greater than $d$. That means the intersection is in the state of idle. In this case, we propose a simple rule to distribute the "right of way" of using the intersection. The nearest one obtains it authorization.

By keeping the arrival order of vehicles, the approach we propose is the following:

\section{Control approach}

1. A vehicle has the green if one of these conditions is satisfied:

(a) If the vehicle is the first one at the intersection, it is allowed to pass through the intersection.

(b) If there is a vehicle of the same road close behind the vehicle passing the intersection $(\leq d)$, this follower receives the green of the precedent vehicle.

(c) If the both following conditions are satisfied:

i. there is not any conflict with all already authorized vehicles,

ii. the vehicle is nearer to the conflict zone than the last authorized vehicle (follower).

2. Otherwise, the last authorized vehicle leaving the intersection sends the green to the nearest vehicle. If there are two vehicles with the same temporal distance, the green is sent to the one that belongs to the same road.

3. Otherwise, the vehicle has red. 
Remark 4. We note that the given control approach:

- does not need much time in computation. Indeed, it is not required to wait for searching information about all vehicles in the intersection before deciding the sequence of their access.

- is close to the well-known "clearing policy". In the remainder of this paper we call this control approach: Distributed Clearing Policy (DCP).

\subsubsection{Example}

Now we give the following example which is depicted in Figure 8 in order to illustrate the control process.

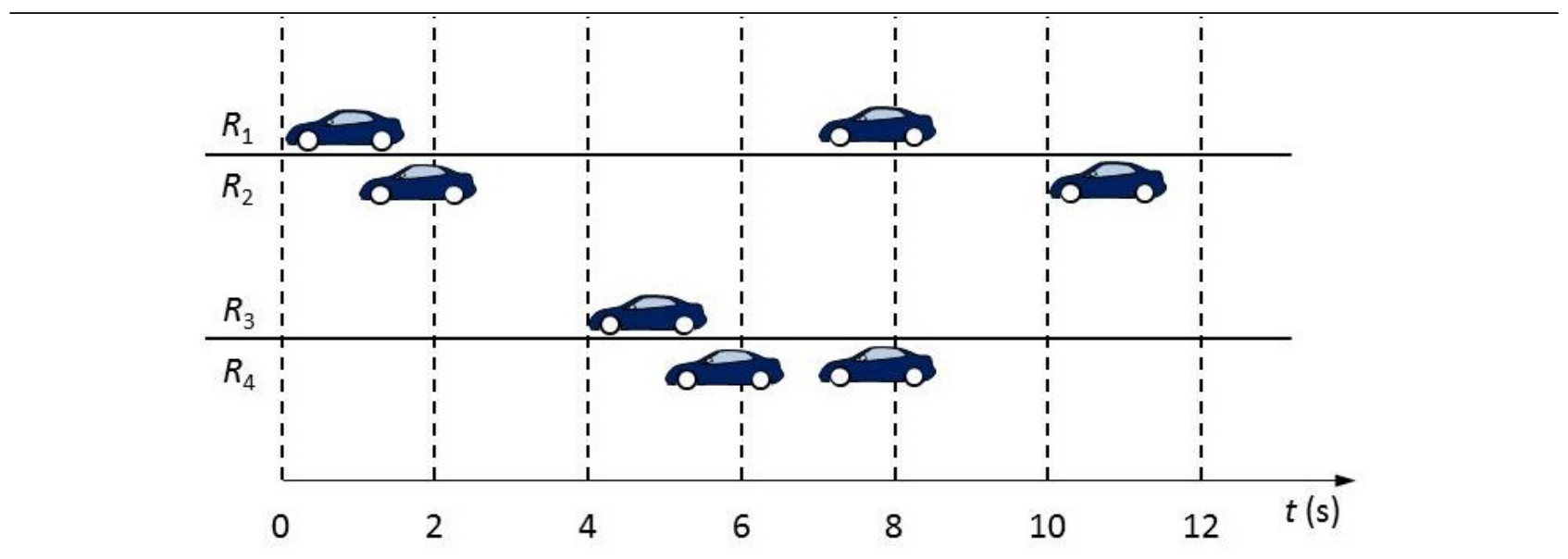

Figure 8: The arrival times

There are two vehicles in road $R_{1}$, two vehicles in road $R_{2}$, one vehicle in road $R_{3}$ and two vehicles in road $R_{4}$. The goal of the control is to form a sequence for crossing the intersection. This sequence has to instantly reduce the queue length. In this example, $d=2 s$ and $S=3 s$.

The first vehicle of $R_{1}$ crosses the intersection because it is the first one. The vehicle that moves in $R_{2}$ receives the "right of way" because it is the nearest to the conflict zone. For the same reason the vehicle that moves in $R_{3}$ gets the "right of way" as well as vehicles that move in $R_{4}$ because they are a team. Afterward, the reminder of vehicles that move in $R_{1}$ and $R_{2}$ cross the intersection without any conflict. The simulation of the sequence is given in Figure 9.

One can check that the sequence given in Figure 9 is the best solution for evacuating all vehicles as soon as possible. However, the second vehicle in $R_{4}$ raises the symmetry issue (see Remark 3), since there is no another vehicle ready to traverse the intersection at $t=8 \mathrm{~s}$ in $R_{3}$. Suppose now that in $R_{3}$ there is an additional vehicle ready to get through the conflict 


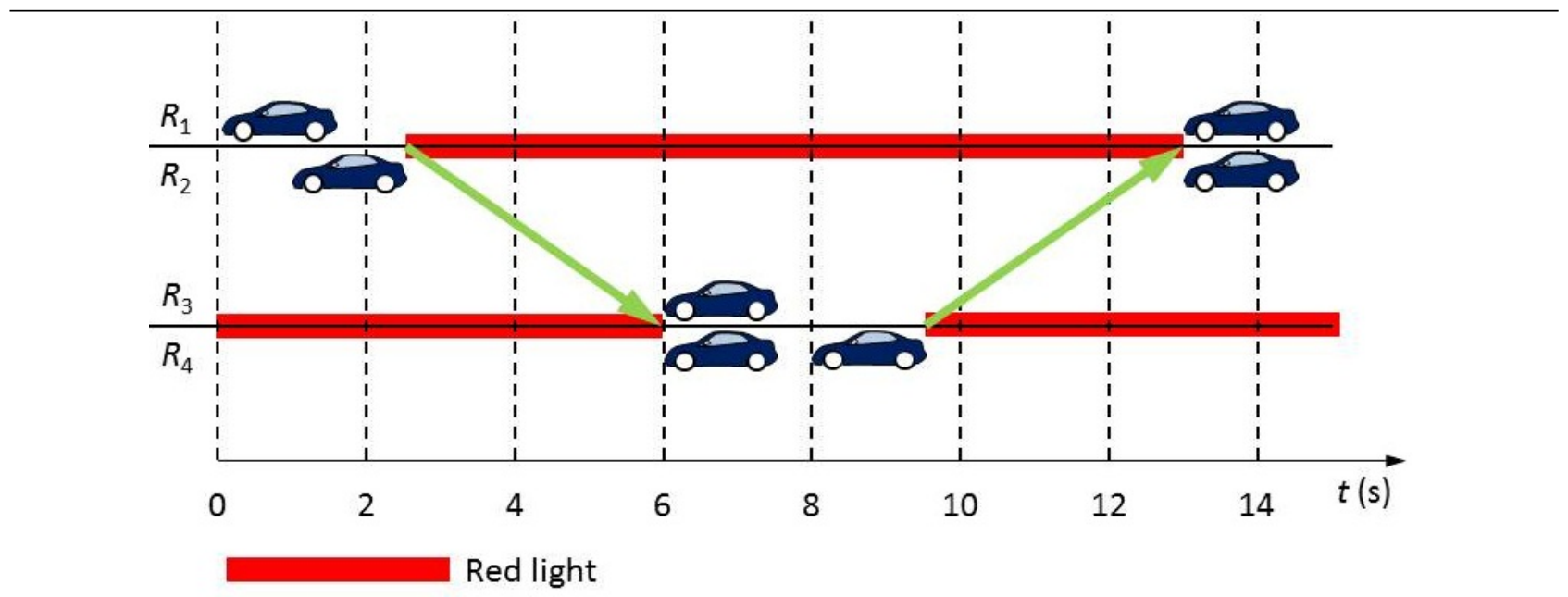

Figure 9: The passing order

zone at $t=14 \mathrm{~s}$. The best solution consists in delaying the "green" given to the second vehicle that moves in $R_{4}$. However, the controller must take a decision in real-time. Indeed, it is not informed about the future arrivals of vehicles. For this reason, we keep the previous rules. In the remainder of the paper, simulations will compare DCP with the optimal sequence that we called "global solution", in which we suppose a controller, informed about the future arrivals of vehicles.

\section{Experiments and simulations}

This section presents experiments of a real cooperative intersection and the simulation results. The experiments has raised the practical constraints which allows to provide simulations as close as possible to reality. Simulation results aim at providing a deep evaluation of DCP by extending the driving environment in terms of intersection layouts and numbers of vehicles.

\subsection{Experiments}

The proposed approach is tested through a real intersection with ordinary vehicles. Before conducting the experiments, a prototype that is an intersection of NXT robots was implemented (Abbas-Turki and al. 2012). A video showing some tests of the experiments with NXT robots and ordinary vehicles is given into Video 1 page 25. The prototype of NXT robots allowed us to highlight the following facts:

- Server: For safety reasons, we have introduced a server through which the vehicles negotiate the "right of way". Without a server, if two conflicting vehicles are not able to 
communicate together, each of them considers that the intersection is free. In simple words, the server allows to set the rule of default deny, whereas, without a server, each vehicle has the green, until it detects the presence of another vehicle.

- Event observer: Due to message delays and losses, certain control approaches, such as First Come First Served (FCFS), can lead to a deadlock. For instance, a follower vehicle receives the "right of way" whereas the precedent has the "red". Hence the server relies on an event observer that allows to avoid such situations (Perronnet et al., 2012).

- Speed limit: The driver must respect a given speed limit before accessing to the storage zone of the intersection. Indeed, the on-board unit must display the red to the driver sufficiently in advance, in order to enable the vehicle to comfortably stop before the conflict zone. The maximal speed is determined according to the accuracy, availability, reliability and latency of the positioning system as well as to the length of the storage zone (between the first and the second position markers in Figure 3).

From these observations, we have considered a server that centralizes the negotiation. We have also determined a speed limit respected by the drivers.

\subsubsection{Intersection}

The experiments were conducted into an industrial zone in Belfort city (Figure 10).

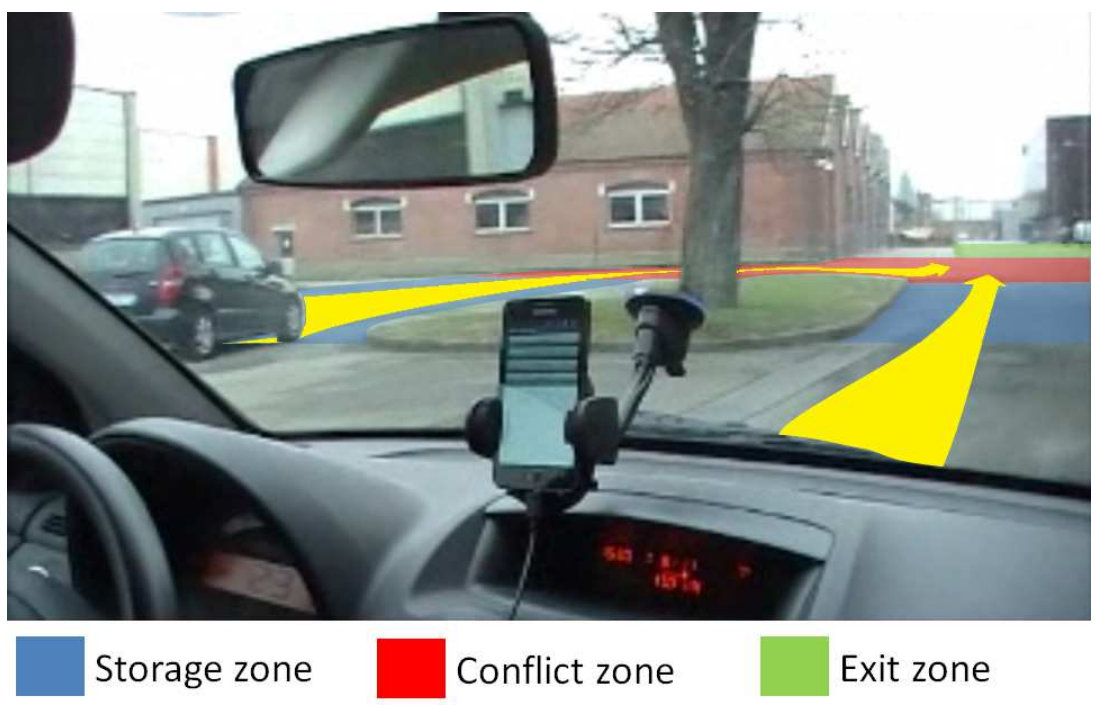

Figure 10: The intersection: Rue Édouard Branly, 90000, Belfort, France 
There is an intersection of two one-way roads. Each road was divided into three zones which are storage, conflict and exit zones. The distance between the beginning of the conflict zone and the end of the exit zone does not exceed $100 \mathrm{~m}$. The intersection is managed through the server that supports the negotiations of the "right of way". Each vehicle has an on-board unit that is able to display the color to the driver, recognize the position of the vehicle and communicate (ITS station) with the server. Instead of using position markers, the intersection zones are identified through the embedded DGPS/EGNOS positioning system. The wireless communication covers a distance of $500 \mathrm{~m}$ without significant problems.

\subsubsection{Vehicle}

Four ordinary vehicles wirelessly negotiate the "right of way" in order to traverse the intersection. The on-board unit displays two colors, i.e. red and green. If the driver observes the red, he must stop before the end of the storage zone. Furthermore, each zone of the intersection determines the behavior of the on-board unit as follows:

- Storage zone:

- display the red,

- launch the process of negotiation: The vehicle sends messages for requesting the "right of way",

- display the green if it receives the "right of way" from the server.

- Conflict zone: check that the vehicle has received the "right of way". Otherwise, send a warning message to the server.

- Exit zone:

- remove the green,

- send an exit request until the reception of a confirmation that the vehicle is removed from the list of the server.

For each vehicle, the default color is "red". This color becomes green only after receiving the "right of way" from the server. The vehicle keeps the green until it leaves the intersection. For safety reasons, the on-board unit displays the red if the driver does not respect the speed limit before getting into the storage zone. This dissuades the drivers from exceeding the speed limit in order to allow them to comfortably brake before the conflict zone. 


\subsubsection{Server}

The main objective of the server is to avoid collisions and deadlocks by analyzing the events. Moreover, the server is in the core of the negotiation process. Hence, in addition to the communication layer, we consider two abstracted layers which are from bottom to top, the "event observer" layer for safety and operation concerns and the "negotiation" layer for ordering the access of vehicles to the conflict zone. The interactions between both layers is first in a bottom-top direction. The event observer feeds the negotiation layer for taking a decision. Second, there is a top-bottom interaction for selecting vehicles that will receive the "right of way". For collision avoidance, the event observer has a clearance list. This list contains the vehicles that have already received the "right of way" but have not accessed yet into the exit zone.

In the experiments, we have tested two approaches for negotiating the "right of way". The first one is FCFS, whereas the second approach is based on our control algorithm. For the first approach, the negotiation layer is a presence list of all vehicles, ordered according to the received requests. In the bottom-top interaction, the event observer removes vehicles that have already received the "right of way" and adds new vehicles. In the top-bottom interaction, the event observer reorders the presence list for avoiding deadlock situations. Then, it selects the first vehicles that will simultaneously receive the "right of way", according to the clearance list.

The second approach which is based on the proposed control algorithm does not require a presence list of all vehicles. For each arrival of vehicle, the decision taken by the negotiation layer depends on the positions of:

- the last vehicle that have received the "right of way",

- the first vehicle waiting for a "right of way".

The decision is then confirmed by the event observer through the clearance list.

\subsubsection{Tests}

The experiments have involved four vehicles. Three drivers are novice users. More precisely, they have discovered the signaling system during the experiments. The experiments have been successfully repeated many times. This allows the novice users to reach a high level of confidence. The drivers have chosen randomly their itinerary. Figure 11 shows an example of a tested sequence.

The protocol has allowed to build the sequences in a real-time application where the detection, the computation and the decision are performed during a very short cycle time less than or equal to $100 \mathrm{~ms}(10 \mathrm{~Hz})$. The first optimization is due to the adaptation of Lemma 1. Indeed, it prevents the server to enumerate all possible states of the intersection. The other optimization is due to the negotiation protocol. It prevents from using a complex algorithm.

In the experiments we have considered two algorithms for distributing the "right of way": FCFS and our control algorithm (DCP). The comparison between the FCFS and DCP shows 


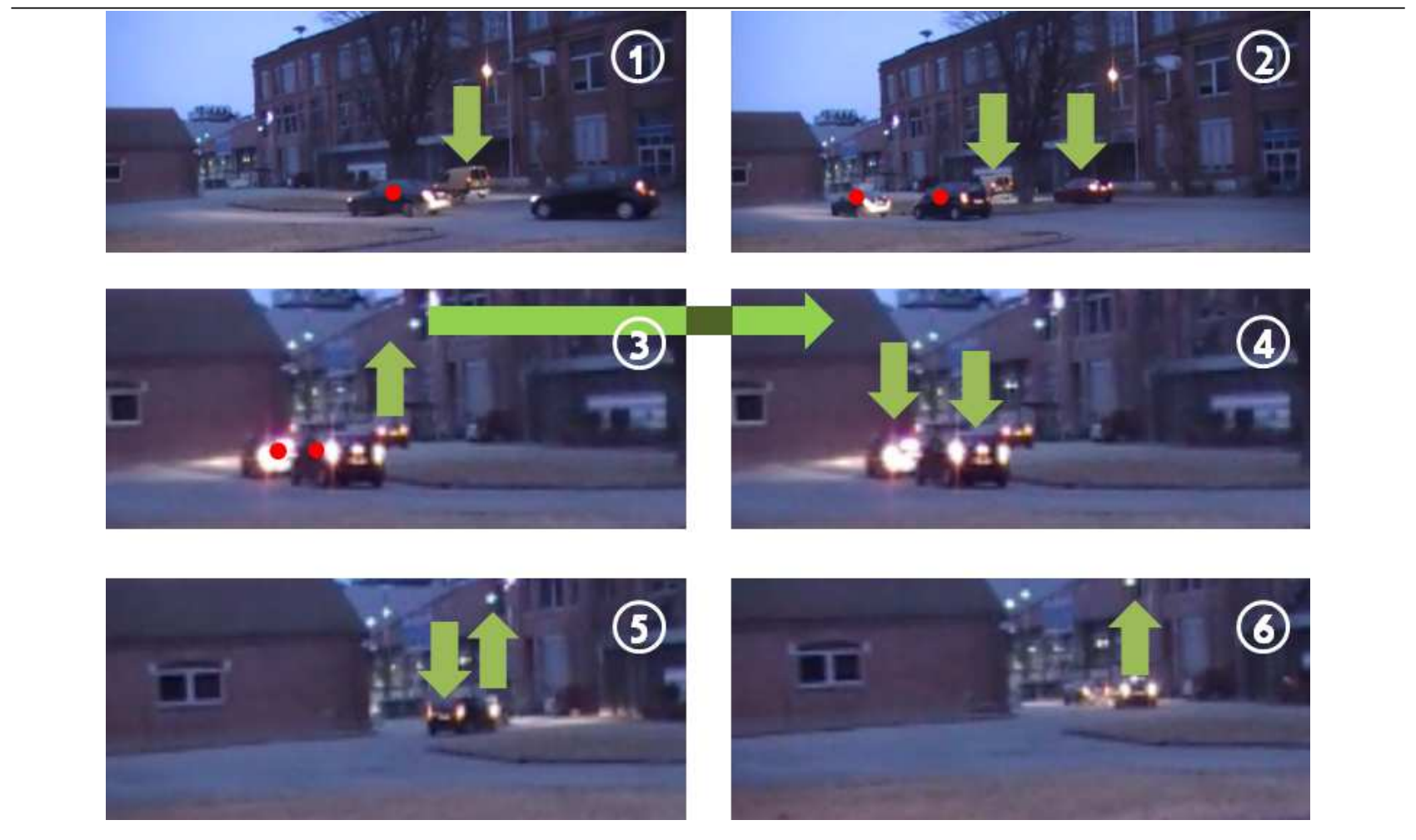

Figure 11: An example of a tested sequence

that time can be saved, even for four vehicles. Except when both approaches provide the same sequence, the control algorithm allows to reduce the evacuation time by minimizing the number of switches. The lost time for switching varies between $2.5 \mathrm{~s}$ and $5 \mathrm{~s}$ whereas two successive vehicles that move in the same road are separated by a temporal distance near to $2 s$. The lost time for switching is due to the distance between the exit zone and the storage zone. The lost time in these experiments is elongated because of the positioning inaccuracies.

Video 1 shows some tests using FCFS and DCP with NXT-robots and ordinary vehicles. They highlight the fact that the lost time for switching is more important than the time required for following another vehicle in the same road. For this reason DCP performs better than FCFS. 


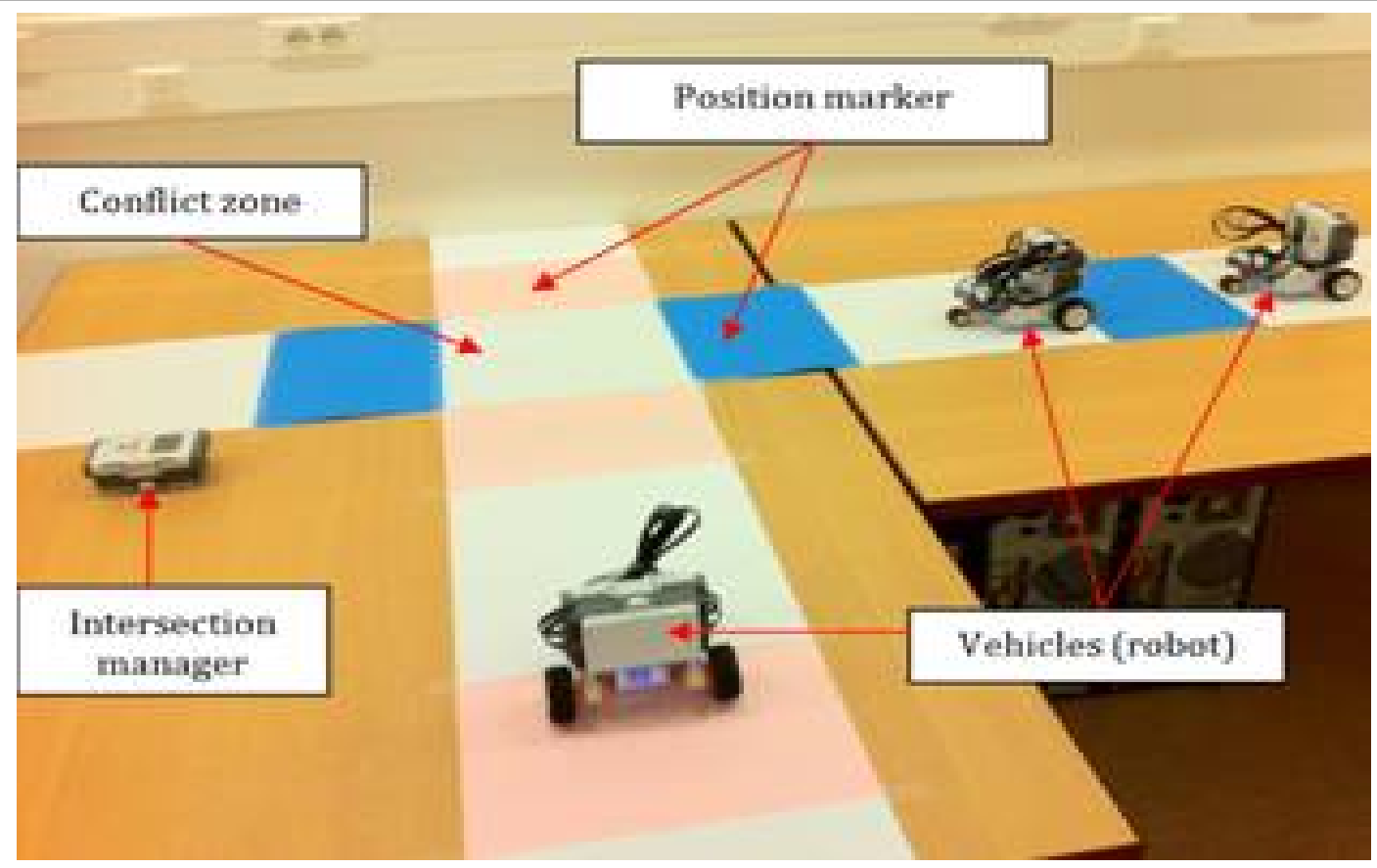

Video 1: Samples of experiments with NXT robots and ordinary vehicles (This video is viewed on http:www.youtube.com/watch?v=EKH34-ce5VM)

\subsection{Simulations}

In the following simulations, we assume that the arrivals of vehicles obey the Poisson distribution (Chen and al., 2010). Since we do not consider the overtaking, the vehicle following model is used during the simulation (Gipps, 1981; Panwai and Dia, 2005). Each vehicle is equipped with a positioning system in order to instantly measure the headway time, as it is required by the proposed heuristic. The safe headway time is about $2 s(d=2 s)$. The lost time is $3 s(S=3 s)$. A value of 3600 vehicles/hour $(1 \mathrm{veh} / \mathrm{s})$ is set as the maximum traffic load (flow rate). The whole simulation framework is based on multiagent system, in order to consider exactly the same architecture of server and on-board units. Nevertheless, we assume that the positioning system is more accurate than the used DGPS/EGNOS system, by assuming the existence of position markers. Two intersections are simulated: the studied simple intersection and an intersection with a more complicated layout. A video showing sample of simulations is given into Video 2 page 31.

\subsubsection{Simple intersection}

Comparisons are made between DCP and the "global solution". The "global solution" is calculated by forward dynamic programming (Wu and al. 2007). First, we simulate the move- 
ments of vehicles during 5 minutes and keep information about all theoretical arrival times of the simulated vehicles $r_{\left(i, q_{i}\right)}$, where $\left(i, q_{i}\right)$ indicates the $q_{i}$ th vehicle arrival on the road $R_{i}$. The goal of the "global solution" aims to find an optimal vehicles passing sequence in order to minimize the maximum exit time of vehicle $e_{\left(i, q_{i}\right)}$. Besides, the time constraints $d$ and $s$ should be respected in the optimal sequence. Hence, the control of the "global solution" consists in resolving the following combinatorial optimization problem (Wu and al., 2011):

\section{Global solution}

- Given: $r_{(i, 1)}, \ldots, r_{\left(i, n_{i}\right)}, i \in[1,4]$ with $r_{\left(i, q_{i}\right)}<r_{\left(i, q_{i}+1\right)}, q_{i} \in\left[1, n_{i}-1\right]$.

- Find $\min \left\{\max _{i \in[1, L]}\left\{e_{\left(i, n_{i}\right)}\right\}\right\}$ subjects to the constraints:

$$
\begin{aligned}
& e_{\left(i, q_{i}+1\right)}-e_{\left(i, q_{i}\right)} \geq d \\
& \left|e_{\left(i, q_{i}\right)}-e_{\left(j, q_{j}\right)}\right| \geq s \text { if } i \neq^{c} j \\
& e_{\left(i, q_{i}\right)} \geq r_{\left(i, q_{i}\right)}
\end{aligned}
$$

where $i \neq \neq^{c} j$ denotes two conflicting movements coming from roads $R_{i}$ and $R_{j}$, and $n_{i}$ denotes the number of vehicles released on the road $R_{i}$ during the 5 minutes.

Then, we apply forward dynamic programming to obtain an optimal solution for solving the above problem. From the description of the "global solution", we can see that it provides the best theoretical sequence of the distribution of the "right of way" to vehicles in order to evacuate all vehicles as soon as possible. This sequence benefits from a theoretical temporal visibility over the time interval of 5 minutes and from more precision than the traditional traffic lights.

Figure 12 and Figure 13 show the waiting time and the average queue length for 5 minutes of simulation at different sums of flow rates, respectively.

The average queue length considers only the stopped vehicles whereas the waiting time is the extra time regarding the theoretical travel time computed without deceleration. Each point in these figures represents the average values of several simulations. Figure 12 and Figure 13 show that the resulting performances from both approaches are close when the traffic flow is low. When the traffic flow is high, a slight distinction can be observed between the two approaches. Indeed, Figure 12 shows that the proposed approach allows to gain at most $2 s$ of the average waiting time whereas Figure 13 shows that the resulting queue lengths are generally worse than the ones resulted from the "global solution". Nevertheless, this loss of performances is limited to at most 2 vehicles. Such a low difference allows us to conclude that the heuristic performs as well as the "global solution". 


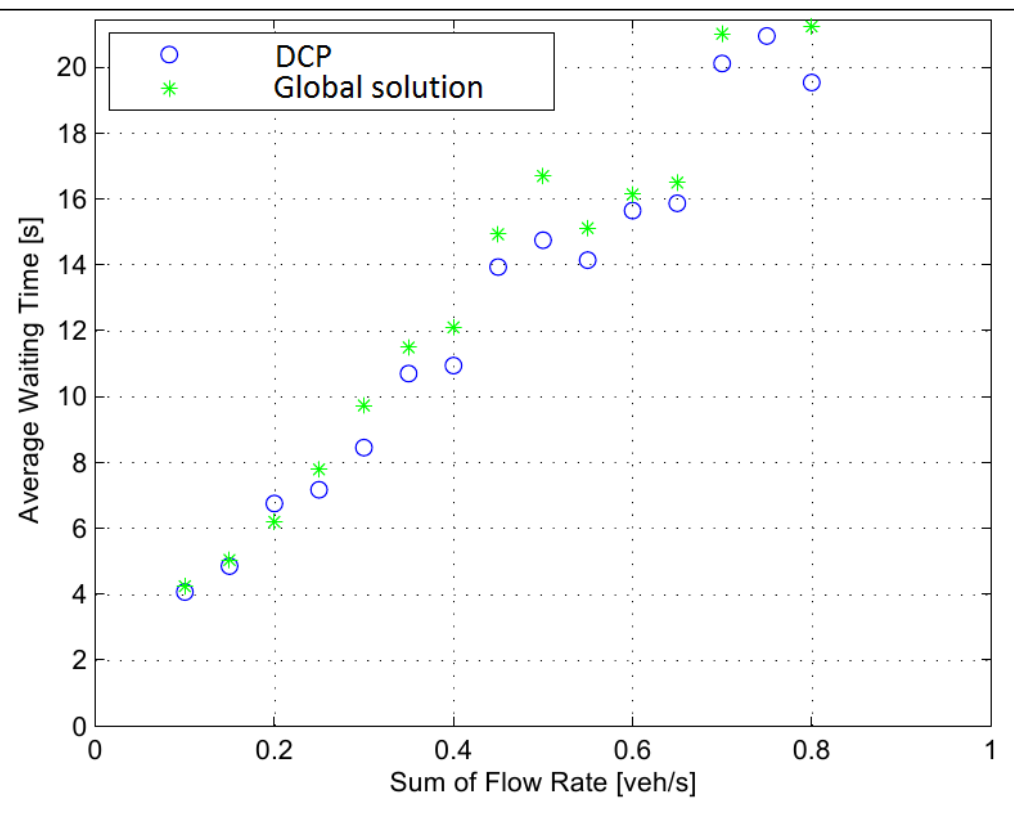

Figure 12: Average waiting time (s)

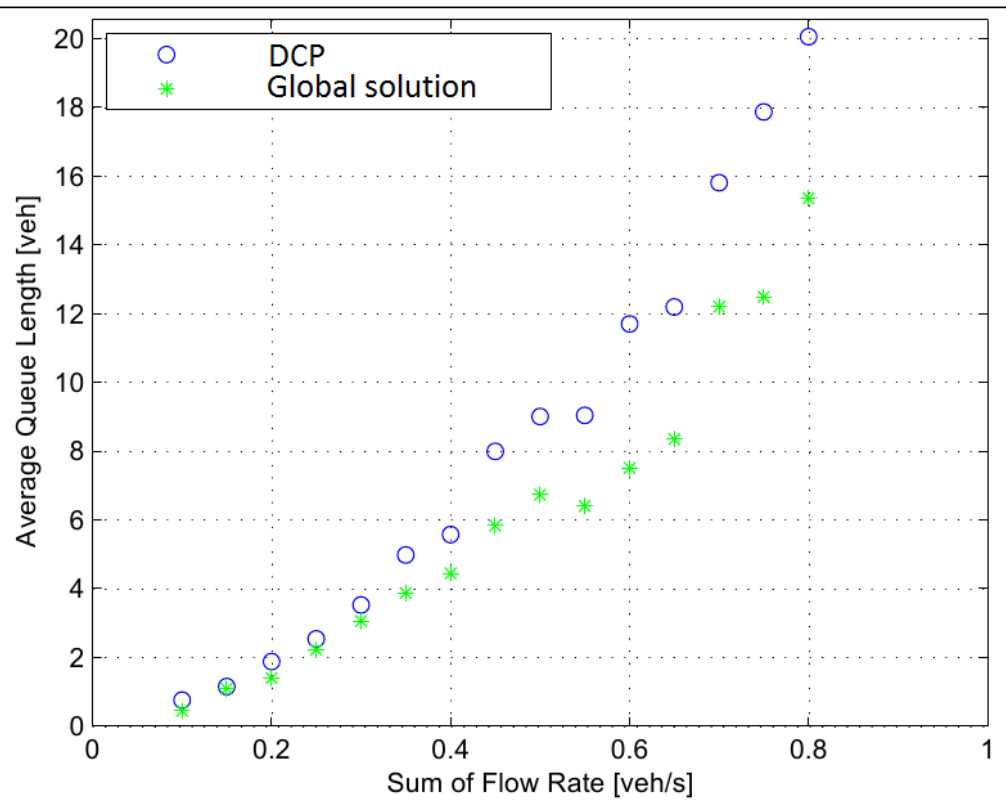

Figure 13: Average queue length (veh) 
This result is due to the following fact: The "global solution" is based on an average behavior of the drivers into the intersection. It is the best theoretical solution based on the arrival times of vehicles detected at the first position marker but the heuristic is efficient because it takes into consideration the data given by the positioning system which allows to instantly measure the headway time. Indeed, the heuristic is based on this real-time detection that is wirelessly communicated during the movement of vehicles independently of the assumed behavior of each vehicle.

\subsubsection{Extended intersection layout}

The layout of the intersection is presented in Figure 14.

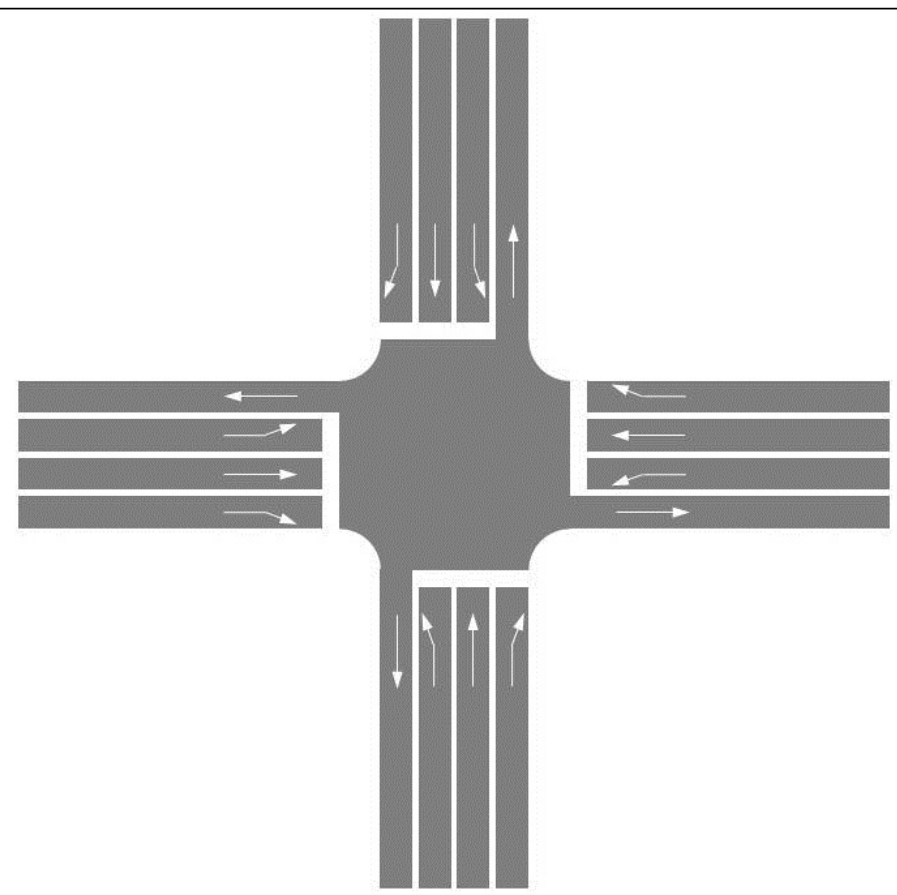

Figure 14: Extended intersection layout

It is a four-arm junction with twelve feeding lanes that correspond to all possible origindestination, including turn-right and turn-left. Our control approach is compared to traffic lights as well as to a memory-less FCFS (ML-FCFS) that is also a cooperative intersection management. As in the precedent simulations, we submit the same sequence of arrival of vehicles to the three control systems. The cycle time of traffic lights is $104 \mathrm{~s}$ and the traffic is uniformly distributed between the twelve lanes.

The ML-FCFS algorithm proceeds as follows: 


\section{Memory-less FCFS}

1. From the vehicle point of view:

(a) priority equals zero,

(b) if the request is not satisfied, add one unit of priority at each new unit of time (1s).

2. From the server point of view:

(a) select the request that has the highest priority,

(b) if there is not any conflict with all already authorized vehicles, the vehicle is allowed to traverse the intersection, otherwise go to (2.a) and keep the request.

As DCP, the ML-FCFS does not require to keep the list of all present vehicles. It just needs to keep the highest priority request. The main objective of introducing the ML-FCFS is to check that the performance of the proposed algorithm is not only due to a real-time distribution of available itineraries.

We have considered two levels of traffic flow which are medium and high. In the medium level, the sum of flow rates is equal to 1800 vehicles/hour whereas in the high level, the cumulative flow rate is equal to 2800 vehicles/hour. The simulation duration is $1000 \mathrm{~s}$. Figure 15 and Figure 16 present a sample of simulations during $1000 \mathrm{~s}$ of medium and high levels of traffic flow, respectively. Both figures, gives the number of vehicles present in each lane at each second.

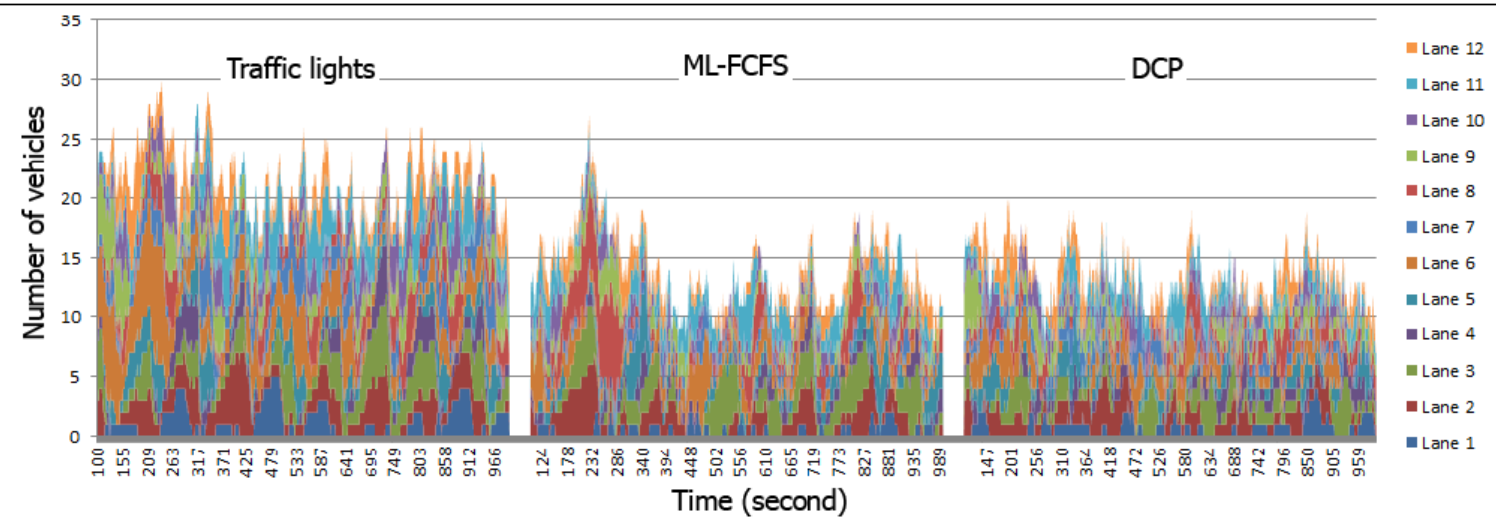

Figure 15: Number of vehicles during $1000 \mathrm{~s}$ for a medium level of traffic

One can observe from both Figures 15 and 16 that DCP has performed better than both other approaches. When the traffic level is medium, the difference between the simulations of ML-FCFS and traffic light is due to the real-time distribution of the available itineraries to vehicles. However, when the traffic becomes denser, this advantage is lost. Indeed, one can observe in Figure 16 that the ML-FCFS quickly diverges whereas the traffic light as well as DCP 


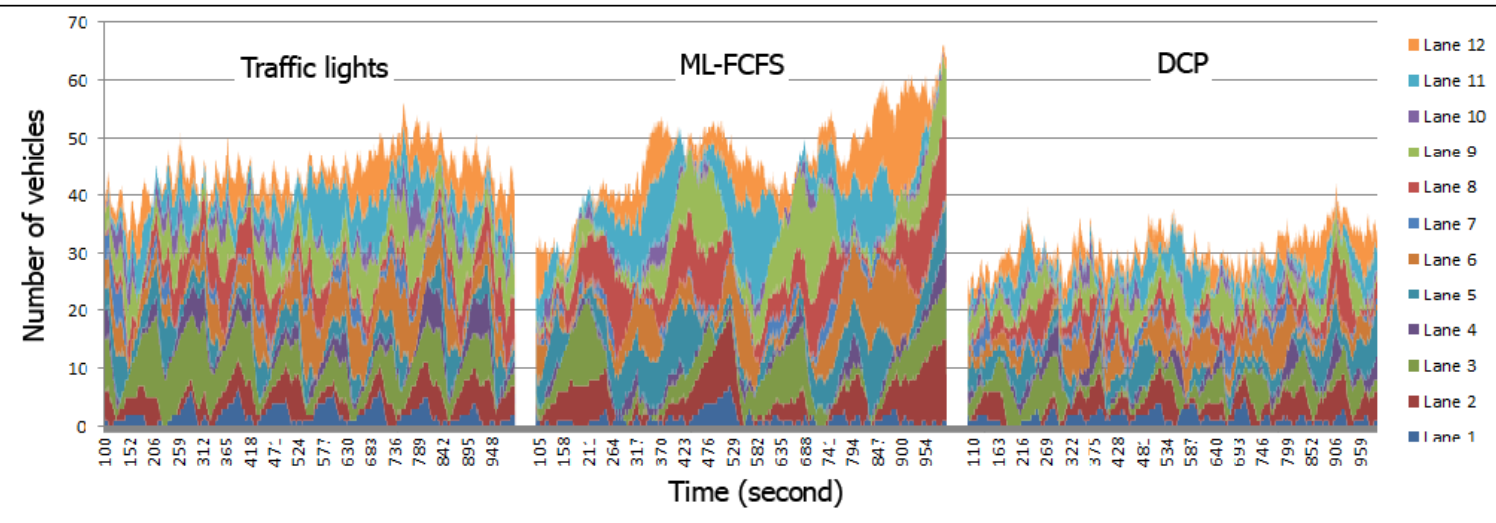

Figure 16: Number of vehicles during 1000s at a high level of traffic

are stable around the average values. Table 4 presents the average stop times resulting from the three approaches after five simulations. The results confirm the advantageous performances of DCP.

\begin{tabular}{|c||c||c||c|}
\hline Control & Traffic lights & ML-FCFS & DCP \\
\hline \hline Medium level & 27.03657 & 14.89467 & 10.37728 \\
\hline High level & 36.26757 & 41.60301 & 21.29748 \\
\hline
\end{tabular}

Table 4: Average stop times (s)

Video 2 provides a sample of simulations. It shows that there is no a big difference between DCP and the global solution whereas it is obvious that DCP performs better than the other control strategies. A sample of simulations of asymmetric traffic is given. It shows that DCP doesn't suffer from the asymmetry. 


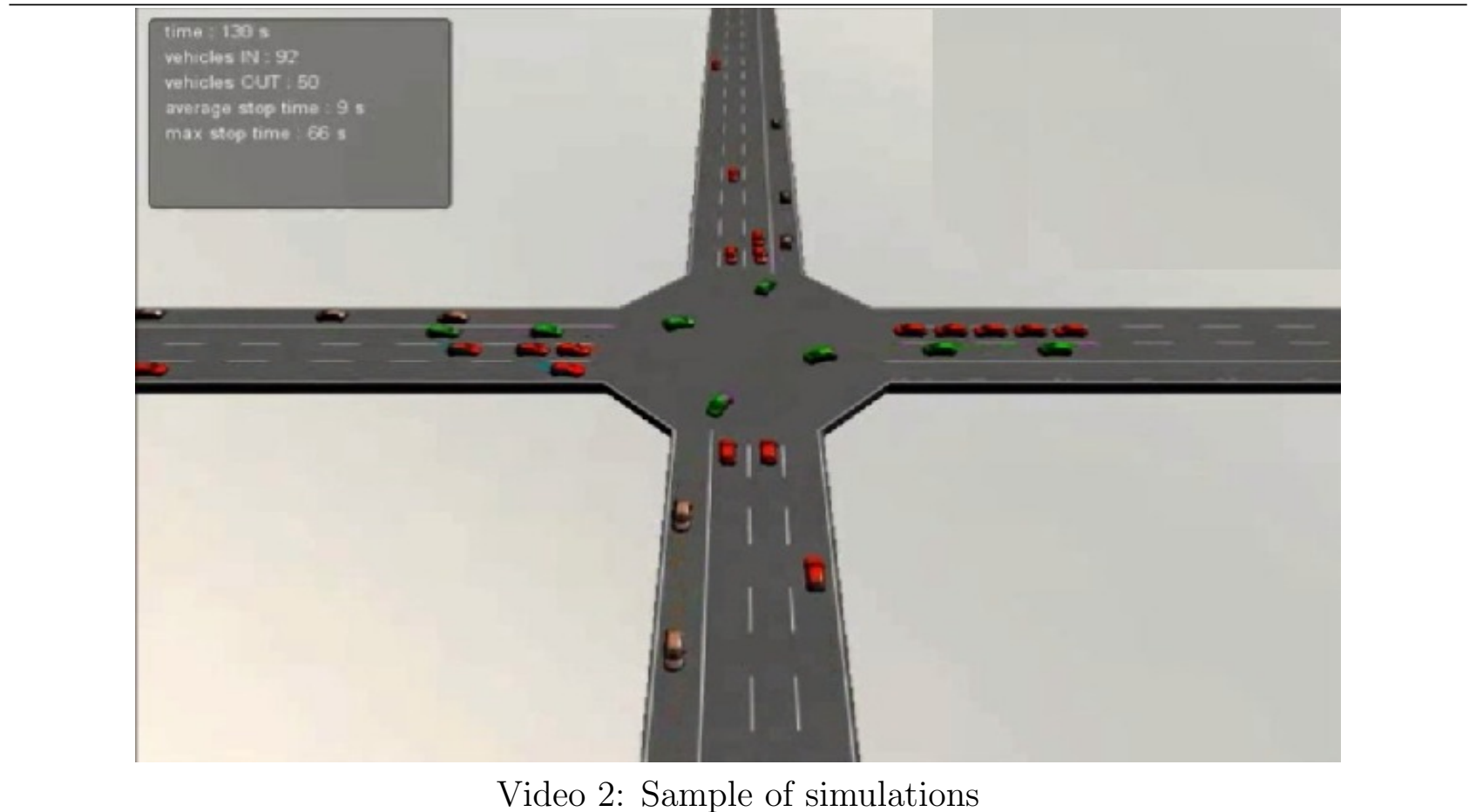

(This video is viewed on http:www.youtube.com/watch?v=ozkfhCR2N9U)

\section{Conclusion}

This paper introduces a new approach for controlling the traffic at isolated intersections. This new traffic control system is able to manage the vehicles individually, through wireless communication and positioning technologies. The "right of way" is displayed to the driver by means of the on-board signalization. Simulations show that the proposed control policy performs as well as the "global solution". The proposed approach is a base for further extensions to a wider system and other considerations, such as a network of intersections or priority roads.

This paper shows that the distributed clearing policy is as efficient as the "classical" scheduling algorithms applied to isolated intersections. However, other rules have to be added if we consider connected intersections. More discussions on the clearing policy are given in (Kumar and Seidman, 1990; Humes, 1994) but the provided solutions deserve further investigations in order to adapt them to the traffic context. The other issue that deserves further investigations is an extension of the switching rules by considering estimated traffic rates. 


\section{References}

Abbas-Turki, A., Ahmane, M., Gao, F., Wu, J., El-Moudni, A., Miraoui, A., 2012. Using Robots for Prototyping Autonomous Intersection Management: Feasibility and Feedbacks. 1st IFAC Conference on Embedded Systems, Computational Intelligence and Telematics in Control, University of Wrzburg, Wrzburg, Germany, pp. 308-313.

Au, T.-C., Quinlan, M., Stone, P., 2012. Setpoint Scheduling for Autonomous Vehicle Controllers. IEEE International Conference on Robotics and Automation, pp. 2055-2060.

Bouyekhf, R., Abbas-Turki, A., Grunder, O., El Moudni, A., 2003. Fluid stochastic Petri net for control of an isolated two-phase intersection. Proceedings of the IEEE Multiconference on Computational Engineering in System Applications, France.

Chard, B.M., Lines, C.J., 1987. Transyt-the latest developments. Traffic engineering and control, Vol. 28, pp. 387-390.

Chen, X., Li, L., Zhang, Y., 2010. A Markov Model for Headway/Spacing Distribution of Road Traffic. IEEE Transaction on Intelligent Transportation Systems, Vol.11, No.4, pp. 773-785.

Contet, J.M., Gechter, F., Gruer, P., Koukam, A., 2007. Physics inspired multiagent system for vehicle platooning. Proceedings of the 6th International Joint Conference on Autonomous Agents and Multiagent Systems, Honolulu, Hawaii, USA, pp. 349-351.

Contet, J.M., Gechter, F., Gruer, P., Koukam, A., 2007. Application of reactive multiagent system to linear vehicle platoon. 19th IEEE International Conference on Tools with Artifical Intelligence, Vol. 2, pp. 67-70.

De La Fortelle, A., 2010. Analysis of reservation algorithms for cooperative planning at intersections. 13th IEEE International Conference on Intelligent Transportation Systems, pp. 445-449.

Di Febbraro, A., Giglio, D., Sacco, N., 2004. Urban traffic control structure based on hybrid Petri nets. IEEE Transactions on Intelligent Transportation Systems, Vol. 5, No. 4, pp. 224-237.

Dotoli, M., Fanti, M.P., 2006. An urban traffic network model via colored timed Petri nets. Control Engineering Practice, Vol. 14, No. 10, pp. 1231-1247. 
Dresner, K.M., Stone, P., 2006. Traffic intersections of the future. Proceedings of the 21st National Conference on Artificial Intelligence, Vol. 2, pp. 1593-1596.

Dresner, K.M., Stone, P., 2004. Multiagent traffic management: a reservation-based intersection control mechanism. Proceedings of the third Inernationale conference on Autonomous Agents and Multiagent Systems, pp. 530-537.

Ferrara, A. , Librino, R., Massola, A., Miglietta, M., Vecchio, C., 2008. Sliding mode control for urban vehicles platooning. IEEE Intelligent Vehicles Symposium, Eindhoven, The Netherlands, pp. 877 - 882.

Gipps, P.G., 1981. A behavioural car following model for computer simulation. Transportation Research Board, Vol.15, No.2, pp. 403-414.

Giua, A., 1991. A traffic light controller based on Petri nets. Rensselaer Polytechnic Institute, Troy, New York.

Grnewald, M., Rust, C., Witkowski, U., 2006. Using mini robots for prototyping intersection management of vehicles. Proceedings of the 3rd International Symposium on Autonomous Minirobots for Research and Edutainment, Part 11, pp. 287-292.

Henry, J.J., Farges, J.L., Tuffal, J., 1983. The prodyn real time traffic algorithm. Proceedings of the 4th IFAC/IFORS Conf. Control Transportation System, pp. 305-309.

Humes, C Jr, 1994. A Regulator Stabilization Technique: KumarSeidman Revisited. IEEE Transactions on Automatic Control, Vol. 39, No. 1, pp. 191-196.

Jingyu, Li, Qiqiang, Li, 2008. Modeling of Urban Traffic System Based on Dynamic Stochastic Fluid Petri Net. Proceedings of the Workshop on Power Electronics and Intelligent Transportation System, pp. 485-491.

Kanaris, A., Ioannou, P., Ho, F.-S., 1997. Spacing and Capacity Evaluations for Different AHS Concepts. Proceedings of the American Control Conference, Albuquerque, New Meixco. pp. 2033-2040.

Kumar P.R, Seidman T.I, 1990. Dynamic Instabilities and Stabilization Methods in Distributed Real-Time Scheduling of Manifacturing Systems. IEEE Transaction On Automatic Control, Vol. 35, No. 3, pp. 289-298. 
Li, L., Wang, F.Y., 2006. Cooperative driving at blind crossings using intervehicle communication. IEEE Transactions on Vehicular technology, Vol. 55, No. 6. pp. 1712-1724.

Motawej, F., Bouyekhf, R., El Moudni, A., 2011. A Dissipativity-Based Approach to Traffic Signal Control for An Over-Saturated Intersection. Journal of the Franklin Institute, Vol. 348, pp. 703-717.

Murata, T., 1989. Petri nets: properties, analysis and applications. Proceeding of the IEEE, Vol. 77 , No. 4, pp. 541-580.

Naumann, R., Rasche, R., Tacken, J., Tahedi, C., 1997. Validation and simulation of a decentralized intersection collision avoidance algorithm. IEEE Conference on Intelligent Transportation System (ITSC), pp. 818-823.

Neuendorf, N., Bruns, T., 2004. The vehicle platoon controller in the decentralised, autonomous intersection management of vehicles. IEEE International Conference on Mechatronics, pp. 375-380.

Papageorgiou, M., Diakaki, C., Dinopoulou, V., Kotsialos, A., Wang, Y., 2003. Review of Road Traffic Control Strategies. Proceedings of the IEEE, Vol. 91, N.12, pp. 2043-2067.

Pan, Y.-J., 2008. Decentralized control of vehicles in platoons with robust nonlinear state estimation. The 4th IEEE Conference on Automation Science and Engineering, Key Bridge Marriott, Washington DC, USA, pp. 145-150.

Panwai, S., Dia, H., 2005. Comparative Evaluation of Microscopic Car-Following Behavior. IEEE Transaction on Intelligent Transportation Systems, Vol.6, No.3, pp. 314 -325 .

Perronnet, F., Abbas-Turki, A., Buisson, J., El Moudni, A., Zo, R., Ahmane, M., 2012. Cooperative Intersection Management: Real Implementation and Feasibility Study of a Sequence Based Protocol for Urban Applications. 15th IEEE Intelligent Transportation Systems Conference, Anchorage, USA, pp. 42 - 47.

Potts, C. N., Kovalyov, M. Y., 2000. Scheduling with batching: A review. European Journal of Operational Research, Vol. 120, No. 2, pp. 228-249. 
Quinlan ,M., Tsz-Chiu, A., Zhu, J., Stiurca, N., Stone, P., 2010. Bringing simulation to life: A mixed reality autonomous intersection. IEEE/RSJ International Conference on Intelligent Robots and Systems, pp. 6083 - 6088.

Robertson, D. I., Bretherton, R., 1991. Optimizing networks of traffic signals in real time-the scoot method. IEEE Transacations on Vehicular Technology, Vol.4, No. 1, pp.11-15.

Sims, A.G., Dobinson, K.W., 1980. The sydney coordinated adaptive traffic(scat) system philosophy and benefits. IEEE Transactions on Vehicular Technology, Vol. 29, No. 2, pp. 130-137.

Gartner, N.H., Pooran, F.J., Andrews, C.M., 2001. Implementation of the opac adaptive control strategy in a traffic signal network. IEEE Intelligent Transportation Systems, pp. 195-200.

Takikita, M., Goto, Y., 2008. DSRC On-board Unit for Multiple Services. Technical Reports, Mitsubishi Electric Advance, march, pp. 17-18.

Wu, J., Abbas-Turki, A., Corra, A., El-Moudni, A., 2007. Discrete Intersection Signal Control. IEEE International Conference on Service Operations and Logistics, and Informatics, pp. 27-29.

Wu, J., A. Abbas-Turki, El-Moudni, A., 2011. Cooperative Driving: An Ant Colony System for Autonomous Intersection Management. Journal of Applied Intelligence, pp.1-16.

Yan, F., Dridi, M., El Moudni, A., 2009. Autonomous Vehicle Sequencing Algorithm at Isolated Intersections. 12th IEEE International Conference on Intelligent Transportation Systems, pp. 1-6. 